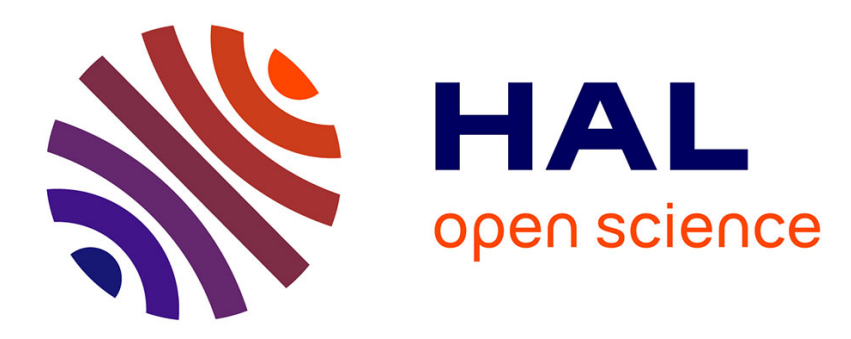

\title{
Topological maps and robust hierarchical Euclidean skeletons in cubical complexes
}

\author{
Michel Couprie
}

\section{To cite this version:}

Michel Couprie. Topological maps and robust hierarchical Euclidean skeletons in cubical complexes. Computer Vision and Image Understanding, 2013, 117 (4), pp.355-369. hal-00828467

\section{HAL Id: hal-00828467 \\ https://hal.science/hal-00828467}

Submitted on 31 May 2013

HAL is a multi-disciplinary open access archive for the deposit and dissemination of scientific research documents, whether they are published or not. The documents may come from teaching and research institutions in France or abroad, or from public or private research centers.
L'archive ouverte pluridisciplinaire HAL, est destinée au dépôt et à la diffusion de documents scientifiques de niveau recherche, publiés ou non, émanant des établissements d'enseignement et de recherche français ou étrangers, des laboratoires publics ou privés. 


\title{
Topological maps and robust hierarchical Euclidean skeletons in cubical complexes
}

\author{
Michel Couprie \\ Université Paris-Est, Laboratoire d'informatique Gaspard-Monge, Équipe \\ A3SI, ESIEE Paris, France. \\ E-mail: m.couprie@esiee.fr
}

\begin{abstract}
Skeletons are notoriously sensitive to contour noise, and an effective filtering scheme is needed in any practical situation where skeletons are involved. In this article, we introduce a new discrete framework that allows us to define and compute families of filtered Euclidean skeletons, in 2D as well as in 3D or higher dimensions. We prove several properties of our skeletonization scheme, in particular the preservation of topological characteristics and the stability with respect to parameter changes. ${ }^{1}$
\end{abstract}

Keywords: skeleton, medial axis, pruning, Euclidean distance, topology preservation, topological map, cubical complex, collapse, stability

Skeleton is one of the most studied and used concepts in pattern recognition and analysis. Since its introduction by H. Blum in the sixties [10], it has been the subject of hundreds of publications dealing with both practical and theoretical aspects. Indeed, despite the simplicity of its most common definition, as the set of all centers of maximal included balls, its use in real applications often raises difficult problems.

These difficulties are mainly due to two distinct issues.

First, the nice properties of skeleton that can be proved in the continuous framework (uniqueness, thinness, homotopy equivalence, invariance w.r.t. isometries) $[28,25]$ do not all hold in discrete grids which are commonly used in image processing. Considerable effort has been devoted to design discrete skeletonization methods that aim at retrieving these properties, at least partially. These methods find their roots in different frameworks: discrete geometry [11, 23, 27, 32, 24], digital topology [19, 40, 39, 31], mathematical morphology [33, 37], computational geometry [2, 3, 29], and partial differential equations [35]. Recent surveys of the state of the art in skeletonization may be found in $[17,36,8,9]$.

\footnotetext{
${ }^{1}$ This work has been partially supported by the "ANR-BLAN07-2_184378 MicroFiss" project and the "ANR-2010-BLAN-0205 Kidico" project.
}

Preprint submitted to Elsevier

November 27, 2012 
Second, even in the continuous framework the skeleton suffers from its sensitivity to small contour perturbations, in other words, its lack of stability. A recent survey [1] summarizes selected relevant studies dealing with this topic. This difficulty can be expressed mathematically: the transformation which associates a shape to its skeleton is only semi-continuous. This fact, among others, explains why it is usually necessary to add a filtering step (or pruning step) to any method that aims at computing the skeleton. Hence, there is a rich literature devoted to skeleton pruning, in which different criteria were proposed in order to discard "spurious" skeleton points or branches: see [4, 29, 3, 27, 2, 38, 24, 5, 18, 26], to cite only a few.

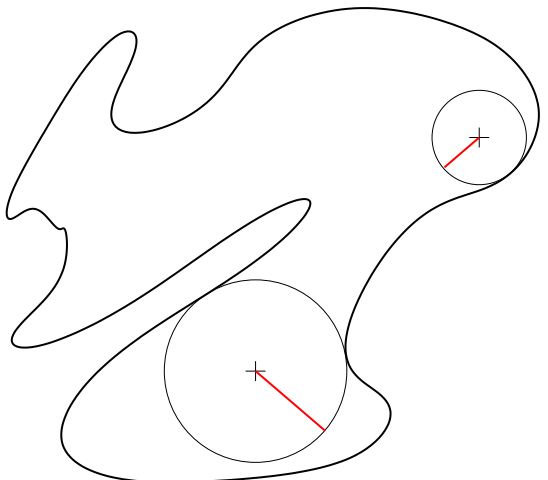

(a)

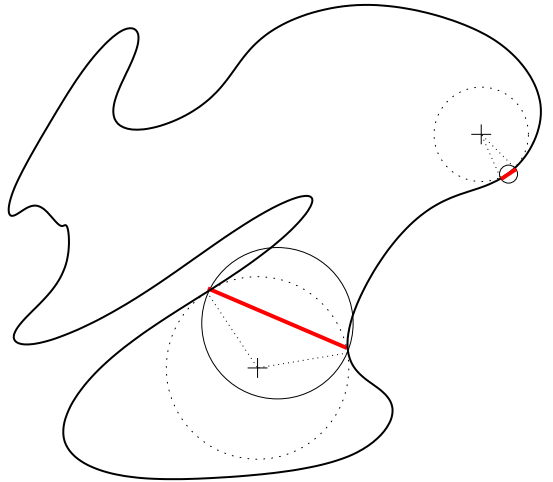

(c)

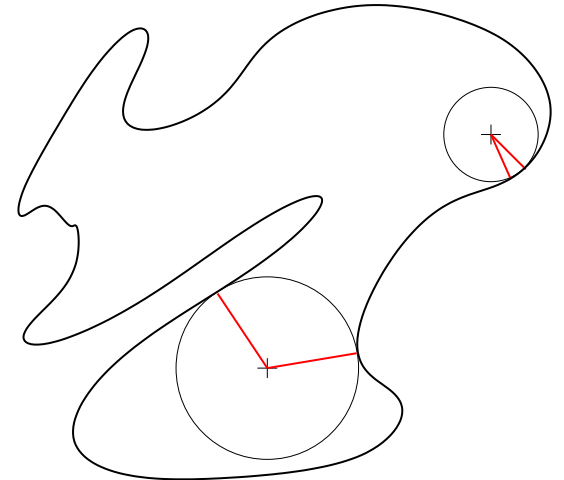

(b)

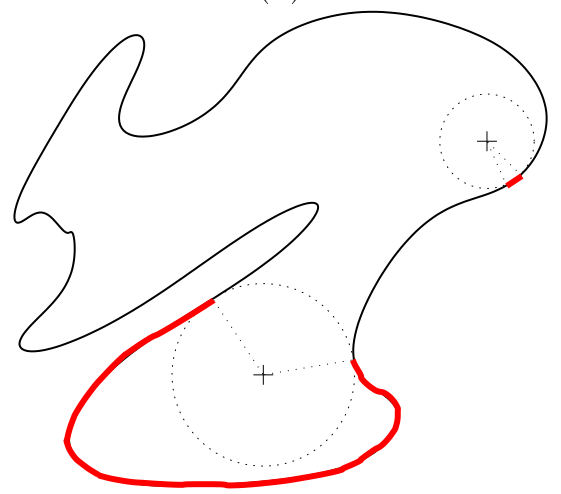

(d)

Figure 1: Four criteria for filtering skeleton points: (a) radius, (b) bisector angle, (c) projection diameter, (d) border portion length.

Fig. 1 illustrates the four most popular ones among these criteria. Consider a skeleton point and its corresponding maximal ball (or disc in 2D), the most obvious criterion is based on the radius of this ball (a): the skeleton point is filtered out if this radius is beyond a given threshold. For defining the second criterion (b) and the following ones, we have to consider the projections of the 
skeleton point on the object boundary, that is, the contact points between the corresponding maximal ball and the boundary. The angle formed by these projections and the skeleton point as vertex, called bisector angle by some authors, also constitutes an effective filtering criterion [39, 18].

If we consider now the distance between the projected points, when there are only two of them, or more generally the diameter ${ }^{1}$ of the smallest ball that contains all these points (see Fig. 1(c)), we obtain the parameter $\lambda$ studied by F. Chazal and A. Lieutier [14], which has interesting properties in relation with stability. These authors introduced a particular class of filtered skeletons, called $\lambda$-medial axes, and they proved that small perturbations (in the sense of the Hausdorff distance) of the shape provoke only small perturbations of the skeleton, except for some critical values of $\lambda$. A discrete version of the $\lambda$-medial axis has been introduced and studied in [13], where its robustness to noise and its low sensitivity to rotations have been shown experimentally.

However, there are applications where the presence of the critical values of $\lambda$ is prohibitive. It is the case when the needed filtering level is equal to, or close to a critical value. In such situations, small changes of the filtering parameter may result in changes of the topological characteristics (e.g. the connectedness), or in sudden elimination or apparition of skeleton branches.

Let us illustrate this problem with the help of Fig. 2. In Fig. 2(a), we see that the parameter value $\lambda=2$ is not sufficient to filter out spurious branches of the $\lambda$-medial axis. However if we set $\lambda=3$, we loose a big and meaningful skeleton branch, whereas some spurious branches are still present.

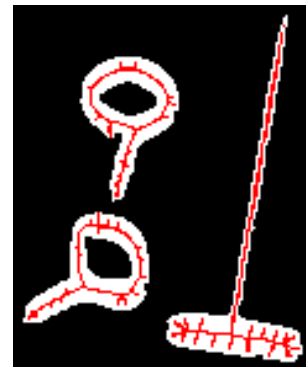

(a)

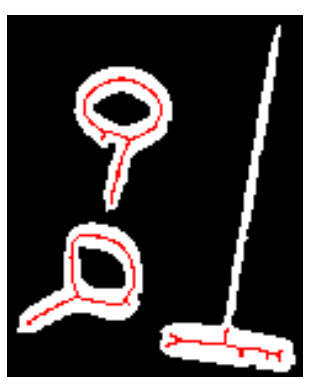

(b)

Figure 2: (a) A shape and its $\lambda$-medial axis, with $\lambda=2$. (b) Idem, with $\lambda=3$.

In 2D, this problem may be avoided by using a fourth criterion, which consists of measuring the length of the portion of the object boundary between the projected points, as illustrated in Fig. 1(d). Based on this idea, several methods have been proposed: hierarchic skeletons [29], veinerization [30], multiscale skeletons [21]. The parameter for these methods is a threshold value for the border portion length criterion. It can be easily seen that small variations of

\footnotetext{
${ }^{1}$ Equivalently, one can consider the radius instead of the diameter.
} 
this parameter do not provoke big changes in the obtained result, contrarily to what happens with the parameter $\lambda$.

Using any of these four criteria, one obtains for any object a family of nested skeletons, indexed by parameter values. Another way of seeing this family, is to consider the function that associates, to each object point, the value of the considered criterion. For example, the function on which is based the $\lambda$-medial axis is called $P R$ (for Projection Radius) in this article. Final skeletons are obtained as level sets (i.e., thresholds) of this function (see Fig. 6).

The aim of this article is to formalize and generalize, in a discrete framework, the approaches based on the fourth criterion (border portion length), for they provide the best stability with respect to variations of the filtering parameter. The method of R.L. Ogniewicz and O. Kübler [29] is defined in the framework of the $2 \mathrm{D}$ constinuous plane, more precisely it applies to (sets of) planar polygons, and the resulting skeletons are made of straight line segments. These skeletons are proved to be homotopy-equivalent with inital objects, however if one needs to discretize these skeletons in $\mathbb{Z}^{2}$, one looses this property. On the other hand, the methods proposed by M. Pierrot-Deseilligny et al. [30] and A.X. Falcao et al. [21] are defined in the 2D square grid. However [30] does not provide an algorithm to compute skeletons in practice, and the algorithm proposed in [21] does not guarantee topology preservation. To the best of our knowledge, these methods have not been extended to the 3D space until now.

The discrete objects that we consider in this article are cubical complexes, that is, they are sets of elements of different dimensions (points, segments, squares, cubes, etc) that are glued together according to certain rules (see Sec. 1). We consider here 2D and 3D cubical spaces, however our approach extends easily to any finite dimension.

The first step of our skeletonization scheme consists of a directional parallel thinning (Sec. 4), guided by the priority function $P R$ (Sec. 3), and based on the operation of collapse (Sec. 2). Collapse is an elementary topology-preserving transformation which is a discrete analog of a continuous deformation (a homotopy). From the collapse sequence produced by this step, we derive an acyclic graph, which we call a flow graph (Sec. 4).

In Sec. 6, we introduce the notion of topological map (based on a flow graph). A topological map is a function, defined on the elements of a cubical complex, that is a particular case of a discrete Morse function [22]. We show that any threshold of a topological map derived from an object $X$ has the same topology as $X$ (Th. 12).

In Sec. 7 we propose a method for computing topological maps that are based on different measures of shape characteristics, such that the one of border portion length. In fact, any conceivable measure may be used at this step, we indicate several meaningful examples. The validity of this method is established by Prop. 17 .

Our new skeletonizon scheme consists of computing a topological map $M$, using the tools described above, and thesholding it at any desired level. We prove the property that, for threshold values that are close to each other, the resulting 
filtered skeletons are also close to each other ${ }^{2}$, with respect to the Hausdorff distance (Th. 14). This property establishes the stability of our method w.r.t. the parameter value.

In Sec. 9, we give some experimental results and comparisons with other methods of the same class. Unlike former approaches to define and compute hierarchic or multiscale skeletons, our method also applies to 3D objects for obtaining curvilinear skeletons (Sec. 10).

\section{Cubical complexes}

In this section, we recall briefly some basic definitions on cubical complexes, see also $[7,6]$ for more details. We consider here $n$-dimensional complexes, mainly with $0 \leqslant n \leqslant 3$.

Let $S$ be a set. If $T$ is a subset of $S$, we write $T \subseteq S$. We denote by $|S|$ the number of elements of $S$.

Let $\mathbb{Z}$ be the set of integers. We consider the families of sets $\mathbb{F}_{0}^{1}, \mathbb{F}_{1}^{1}$, such that $\mathbb{F}_{0}^{1}=\{\{a\} \mid a \in \mathbb{Z}\}, \mathbb{F}_{1}^{1}=\{\{a, a+1\} \mid a \in \mathbb{Z}\}$. A subset $f$ of $\mathbb{Z}^{n}, n \geqslant 2$, which is the Cartesian product of exactly $m$ elements of $\mathbb{F}_{1}^{1}$ and $(n-m)$ elements of $\mathbb{F}_{0}^{1}$ is called a face or an $m$-face in $\mathbb{Z}^{n}, m$ is the dimension of $f$, we write $\operatorname{dim}(f)=m$.

Observe that any non-empty intersection of faces is a face. For example, the intersection of two 2-faces $A$ and $B$ may be either a 2 -face (if $A=B$ ), a 1-face, a 0 -face, or the empty set.

We denote by $\mathbb{F}^{n}$ the set composed of all faces in $\mathbb{Z}^{n}$. An $m$-face is called a point if $m=0$, a (unit) edge if $m=1$, a (unit) square if $m=2$, a (unit) cube if $m=3$.

Let $f$ be a face in $\mathbb{F}^{n}$. We set $\hat{f}=\left\{g \in \mathbb{F}^{n} \mid g \subseteq f\right\}$ and $\hat{f}^{*}=\hat{f} \backslash\{f\}$.

Any $g \in \hat{f}$ is called a face of $f$.

We call star of $f$ the set $\check{f}=\left\{g \in \mathbb{F}^{n} \mid f \subseteq g\right\}$, and we write $\check{f}^{*}=\check{f} \backslash\{f\}$ : any element of $\check{f}$ is a coface of $f$. It is plain that $g \in \hat{f}$ iff $f \in \check{g}$.

A finite set $X$ of faces in $\mathbb{F}^{n}$ is a complex (in $\mathbb{F}^{n}$ ) if for each face $f \in X$, we have $\hat{f} \subseteq X$. See in Fig. 3(d) an example of a complex, and in Fig. 3(b,c) examples of sets of faces that are not complexes.

\section{Collapse}

The collapse operation is an elementary topology-preserving transformation which has been introduced by J.H.C. Whitehead [41] and plays an important role in combinatorial topology. It can be seen as a discrete analogue of a continuous deformation (a strong deformation retract). Collapse is known to preserve the homotopy type.

\footnotetext{
${ }^{2}$ in the sense of Lipschitz continuity
} 


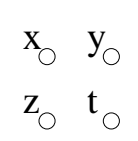

(a)

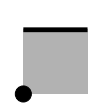

(b)

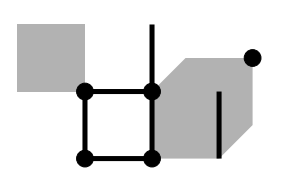

(c)

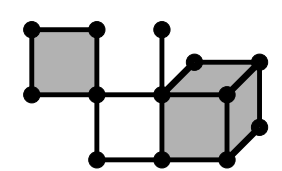

(d)

Figure 3: (a) Four points in $\mathbb{Z}^{2}: x=(0,1) ; y=(1,1) ; z=(0,0) ; t=(1,0)$. (b) A graphical representation of the set of faces $\left\{f_{0}, f_{1}, f_{2}\right\}$, where $f_{0}=\{z\}=\{0\} \times\{0\}$ (a 0-face), $f_{1}=\{x, y\}=\{0,1\} \times\{1\}$ (a 1-face), and $f_{2}=\{x, y, z, t\}=\{0,1\} \times\{0,1\}$ (a 2-face). (c) A set of faces that is not a complex. (d) A set of faces that is a complex.

Let $X$ be a complex in $\mathbb{F}^{n}$ and let $(f, g) \in X^{2}$. If $f$ is the only face of $X$ that strictly includes $g$, then $g$ is said to be free for $X$ and the pair $(f, g)$ is said to be a free pair for $X$. In other terms, $(f, g)$ is a free pair for $X$ whenever $\check{g}^{*} \cap X=\{f\}$. Notice that, if $(f, g)$ is a free pair, then we have necessarily $\operatorname{dim}(g)=\operatorname{dim}(f)-1$.

Let $X$ be a complex, and let $(f, g)$ be a free pair for $X$. Let $m=\operatorname{dim}(f)$. The complex $X \backslash\{f, g\}$ is an elementary collapse of $X$, or an elementary $m$ collapse of $X$.

Let $X, Y$ be two complexes. We say that $X$ collapses onto $Y$, and we write $X \searrow Y$, if $Y=X$ or if there exists a collapse sequence from $X$ to $Y$, i.e., a sequence of complexes $\left\langle X_{0}, \ldots, X_{\ell}\right\rangle$ such that $X_{0}=X, X_{\ell}=Y$, and $X_{i}$ is an elementary collapse of $X_{i-1}$, for each $i \in\{1, \ldots, \ell\}$. See Fig. 4 for an illustration. Let $J=\left\langle\left(f_{i}, g_{i}\right)\right\rangle_{i=1}^{\ell}$ be the sequence of pairs of faces of $X$ such that $X_{i}=X_{i-1} \backslash\left\{f_{i}, g_{i}\right\}$, for any $i \in\{1, \ldots, \ell\}$. We also call the sequence $J$ a collapse sequence (from $X$ to $Y$ ).

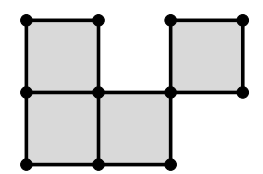

$X$

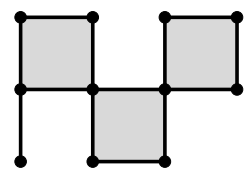

$X_{1}$

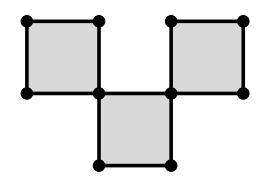

$X_{2}$

Figure 4: $X$ : a 2-dimensional complex. $X_{1}$ : a complex such that $X$ collapses onto $X_{1}$; a free pair composed of a square and an edge has been removed. $X_{2}$ : a complex such that $X_{1}$ collapses onto $X_{2}$; (a free pair composed of an edge and a vertex has been removed), hence $X$ collapses onto $X_{2}$.

Let us now state an elementary property of collapse, which gives a necessary and sufficient condition under which two collapse operations may be performed in parallel (or in any order) while preserving topology.

Proposition 1. Let $X$ be a complex, and let $(f, g)$ and $(k, \ell)$ be two distinct free pairs for $X$. The complex $X$ collapses onto $X \backslash\{f, g, k, \ell\}$ if and only if $f \neq k$. In this case, $\langle(f, g),(k, \ell)\rangle$ and $\langle(k, \ell),(f, g)\rangle$ are both collapse sequences from $X$. 
Proof. If $f=k$, then it is plain that $(k, \ell)$ is not a free pair for $Y=X \backslash\{f, g\}$ as $k=f \notin Y$. Also, $(f, g)$ is not free for $X \backslash\{k, \ell\}$. If $f \neq k$, then we have $g \neq \ell, \check{g}^{*} \cap X=\{f\}(g$ is free for $X)$ and $\check{\ell}^{*} \cap X=\{k\}(\ell$ is free for $X)$. Thus, we have $\check{\ell}^{*} \cap Y=\{k\}$ as $\ell \neq g$ and $k \neq f$. Therefore, $(k, \ell)$ is a free pair for $Y$. The same reasonning shows that $(f, g)$ is a free pair for $Y^{\prime}=X \backslash\{k, \ell\}$.

From Prop. 1, the following corollary is immediate.

Corollary 2. Let $X$ be a complex, and let $\left(f_{1}, g_{1}\right) \ldots\left(f_{m}, g_{m}\right)$ be $m$ distinct free pairs for $X$ such that, for all $a, b \in\{1, \ldots, m\}$ (with $a \neq b), f_{a} \neq f_{b}$. The complex $X$ collapses onto $X \backslash\left\{f_{1}, g_{1} \ldots f_{m}, g_{m}\right\}$.

The corollary (Cor. 4) of the following property will be useful in the sequel.

Proposition 3. Let $J=\left\langle\left(f_{i}, g_{i}\right)\right\rangle_{i=1}^{\ell}$ be a collapse sequence from a complex $X$ to a complex $Y$. Let $j \in\{2, \ldots, \ell\}$ such that $\left(f_{j}, g_{j}\right)$ is free for $X$. Let $J^{\prime}$ denote the sequence obtained from $J$ by swapping pairs $j-1$ and $j$, more precisely, $J^{\prime}=\left\langle\left(f_{i}^{\prime}, g_{i}^{\prime}\right)\right\rangle_{i=1}^{\ell}$ with $f_{j}^{\prime}=f_{j-1}, g_{j}^{\prime}=g_{j-1}, f_{j-1}^{\prime}=f_{j}, g_{j-1}^{\prime}=g_{j}$, and for all $i \in\{1, \ldots, \ell\} \backslash\{j, j-1\}, f_{i}^{\prime}=f_{i}$ and $g_{i}^{\prime}=g_{i}$. Then, the sequence $J^{\prime}$ is also a collapse sequence from $X$ to $Y$.

Proof. We set $X_{i}^{\prime}=X_{i-1}^{\prime} \backslash\left\{f_{i}^{\prime}, g_{i}^{\prime}\right\}$ and $X_{i}=X_{i-1} \backslash\left\{f_{i}, g_{i}\right\}$, for all $i \in\{1, \ldots, \ell\}$, and $X_{0}^{\prime}=X_{0}=X$. Obviously, we have $X_{i}^{\prime}=X_{i}$ for all $i \in\{0, \ldots, \ell\} \backslash\{j-1\}$, thus we only have to prove $X_{j-2} \searrow X_{j-1}^{\prime} \searrow X_{j}$. We know that $\left(f_{j-1}, g_{j-1}\right)$ is free for $X_{j-2}$ (since $J$ is a collapse sequence), and that $\left(f_{j}, g_{j}\right)$ is free for $X_{j-2}$ (since it free for $X$ ), hence $X_{j-2} \searrow X_{j-1}^{\prime}$. Furthermore, $f_{j-1} \neq f_{j}$ because both pairs are in the collapse sequence $J$. By Prop. 1 , we deduce that $\left(f_{j-1}, g_{j-1}\right)$ is free for $X_{j-2} \backslash\left\{f_{j}, g_{j}\right\}=X_{j-1}^{\prime}$, hence $X_{j-1}^{\prime} \searrow X_{j}$.

Corollary 4. Let $J=\left\langle\left(f_{i}, g_{i}\right)\right\rangle_{i=1}^{\ell}$ be a collapse sequence from a complex $X$ to a complex $Y$. Let $j_{1}, \ldots, j_{k} \in\{1, \ldots, \ell\}$ be distinct indices such that $\left(f_{j_{i}}, g_{j_{i}}\right)$ is free for $X$ for any $i \in\{1, \ldots, k\}$. Let $J^{\prime}$ denote the sequence obtained from $J$ by shifting pairs $j_{i}$ to the beginning of the sequence, more precisely, $J^{\prime}=$ $\left\langle\left(f_{i}^{\prime}, g_{i}^{\prime}\right)\right\rangle_{i=1}^{\ell}$ with $f_{i}^{\prime}=f_{j_{i}}, g_{i}^{\prime}=g_{j_{i}}$ for all $i \in\{1, \ldots, k\}$, and the other pairs of $J^{\prime}$ are the remaining pairs of $J$ left in the same order. Then, the sequence $J^{\prime}$ is also a collapse sequence from $X$ to $Y$.

\section{The discrete $\lambda$-medial axis and the projection radius map}

The original definition of the $\lambda$-medial axis (see [14]) holds and makes sense in the (continuous) Euclidean $n$-dimensional space. The definition of a discrete $\lambda$-medial axis (DLMA) in $\mathbb{Z}^{n}$ is given in [13], together with an experimental evaluation of its stability and rotation invariance.

Notice that the DLMA applies on a digital image (i.e., a subset of $\mathbb{Z}^{n}$ ), not on a complex. However, the bijective correspondance between elements of $\mathbb{Z}^{n}$ and $n$-faces in $\mathbb{F}^{n}$ allows us to use the DLMA and related notions in the context of cubical complexes. 
Let $x=\left(x_{1}, \ldots, x_{n}\right), y=\left(y_{1}, \ldots, y_{n}\right) \in \mathbb{R}^{n}$, we denote by $d(x, y)$ the Euclidean distance between $x$ and $y$, in other words, $d(x, y)=\left(\sum_{k=1}^{n}\left(y_{k}-x_{k}\right)^{2}\right)^{\frac{1}{2}}$. Let $S \subseteq \mathbb{R}^{n}$, we set $d(y, S)=\min _{x \in S}\{d(y, x)\}$.

Let $x \in \mathbb{R}^{n}, r \in \mathbb{R}^{+}$, we denote by $B_{r}(x)$ the ball of radius $r$ centered on $x$, defined by $B_{r}(x)=\left\{y \in \mathbb{R}^{n} \mid d(x, y) \leqslant r\right\}$.

Let $S$ be a nonempty subset of $\mathbb{R}^{n}$, and let $x \in \mathbb{R}^{n}$. The projection of $x$ on $S$, denoted by $\Pi_{S}(x)$, is the set of points $y$ of $S$ which are at minimal distance from $x$; more precisely,

$$
\Pi_{S}(x)=\{y \in S \mid \forall z \in S, d(y, x) \leqslant d(z, x)\} .
$$

Let $X$ be an open bounded subset of $\mathbb{R}^{n}$, and let $\lambda \in \mathbb{R}^{+}$. We denote by $\bar{X}$ the complement set of $X$, i.e., $\bar{X}=\mathbb{R}^{n} \backslash X$. The $\lambda$-medial axis of $X$ is the set of points $x$ in $X$ such that the radius of the smallest ball that includes $\Pi_{\bar{X}}(x)$ is not less than $\lambda$ (see Fig. 5).

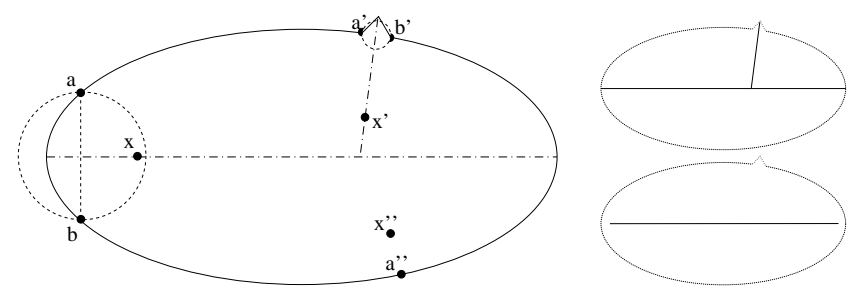

Figure 5: Illustration of the $\lambda$-medial axis. Left: Points $x, x^{\prime}$ and $x^{\prime \prime}$ and their respective closest boundary points. Top right: $\lambda$-medial axis with $\lambda=\epsilon$, a very small positive real number. Bottom right: $\lambda$-medial axis with $\lambda=d\left(a^{\prime}, b^{\prime}\right)+\epsilon$.

For each point $x \in \mathbb{Z}^{n}$, we define the direct neighborhood of $x$ as $N(x)=$ $\left\{y \in \mathbb{Z}^{n} \mid d(x, y) \leqslant 1\right\}$.

Transposing directly the definition of the $\lambda$-medial axis to the discrete grid $\mathbb{Z}^{n}$ would yield unsatisfactory results (see [13]), this is why we need the following notion. Let $S \subseteq \mathbb{Z}^{n}$, and let $x \in S$. The extended projection of $x$ on $\bar{S}$ (where $\left.\bar{S}=\mathbb{Z}^{n} \backslash S\right)$, denoted by $\Pi \frac{e}{S}(x)$, is the union of the sets $\Pi_{\bar{S}}(y)$, for all $y$ in $N(x)$ such that $d(y, \bar{S}) \leqslant d(x, \bar{S})$.

Let $S$ be a finite subset of $\mathbb{Z}^{n}$, and let $\lambda \in \mathbb{R}^{+}$. We define the function $P R_{S}$ which associates, to each point $x$ of $S$, the value $P R_{S}(x)$ that is the radius of the smallest ball enclosing all the points of the extended projection of $x$ on $\bar{S}$. In other terms, $P R_{S}(x)=\min \left\{r \in \mathbb{R}^{+} \mid \exists y \in \mathbb{R}^{n}, B_{r}(y) \supseteq \Pi_{\bar{S}}(x)\right\}$, and we call $P R_{S}(x)$ the projection radius of $x$ (for $S$ ). The discrete $\lambda$-medial axis of $S$, denoted by $D L M A(S, \lambda)$, is the set of points $x$ in $S$ such that $P R_{S}(x) \geqslant \lambda$.

In Fig. 6, we show the function $P R_{S}$ and three examples of DLMAs of a shape $S$. Note that the function $P R_{S}$ can be computed once and stored as a grayscale image, and that any DLMA of $S$ is a level set of this function at a particular value $\lambda$. Notice also that DLMA has not, in general, the same topology as the original shape. For more details, illustrations and performance analysis, see [13]. 


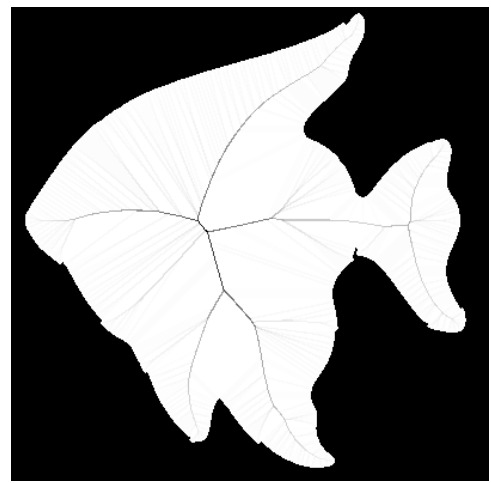

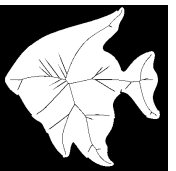

(b)

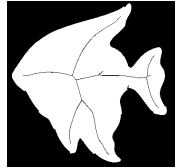

(c)

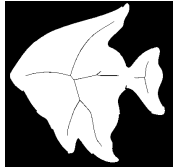

(d)

(a)

Figure 6: (a) The function $P R_{S}$ superimposed to the shape $S$. Darkest colors represent highest values of $P R_{S}(x)$. Any DLMA of $S$ is a level set of this function at a particular value $\lambda$. (b,c,d) Discrete lambda-medial axis with $\lambda=10,30,45$ respectively.

\section{Guided collapse and flow graph}

In this section we introduce a thinning scheme that produces a collapse sequence, based on an arbitrary priority map (e.g., a distance map or a projection radius map). The general idea of guided thinning is not new: it has been used by several authors to produce skeletons based on the Euclidean distance $[19,40,39,31]$, and consists of using the priority function in order to specify which elements must be considered at each step of the thinning. Here, we combine this general idea with a parallel directional collapse algorithm introduced in [12], in order to minimize the number of arbitrary decisions. When several elements share the same priority, which may occur quite often, we remove in parallel all such elements that satisfy a condition based on direction and dimension. All directions and dimensions are successively explored.

First, we need to define the direction of a free face. Let $X$ be a complex in $\mathbb{F}^{n}$, let $(f, g)$ be a free pair for $X$. Since $(f, g)$ is free, we know that $\operatorname{dim}(g)=$ $\operatorname{dim}(f)-1$, and it can be easily seen that $f=g \cup g^{\prime}$ where $g^{\prime}$ is the translate of $g$ by one of the $2 n$ vectors of $\mathbb{Z}^{n}$ with all coordinates equal to 0 except one, which is either +1 or -1 . Let $v$ denote this vector, and $c$ its non-null coordinate. We define $\operatorname{Dir}(f, g)$ as the index of $c$ in $v$, it is the direction of the free pair $(f, g)$. Its orientation is defined as $\operatorname{Orient}(f, g)=1$ if $c=+1$, and as Orient $(f, g)=0$ otherwise.

Considering two distinct free pairs $(f, g)$ and $(i, j)$ for a complex $X$ in $\mathbb{F}^{n}$ such that $\operatorname{Dir}(f, g)=\operatorname{Dir}(i, j)$ and $\operatorname{Orient}(f, g)=\operatorname{Orient}(i, j)$, we have $f \neq i$. From this observation and Cor. 2, we deduce the following property.

Corollary 5. Let $X$ be a complex in $\mathbb{F}^{n}$, and let $\left(f_{1}, g_{1}\right), \ldots,\left(f_{m}, g_{m}\right)$ be $m$ distinct free pairs for $X$ having all the same direction and the same orientation. The complex $X$ collapses onto $X \backslash\left\{f_{1}, g_{1}, \ldots, f_{m}, g_{m}\right\}$. 
Now, we are ready to introduce algorithm 1 . The symbol + is used to denote the action of appending an element at the end of a sequence.

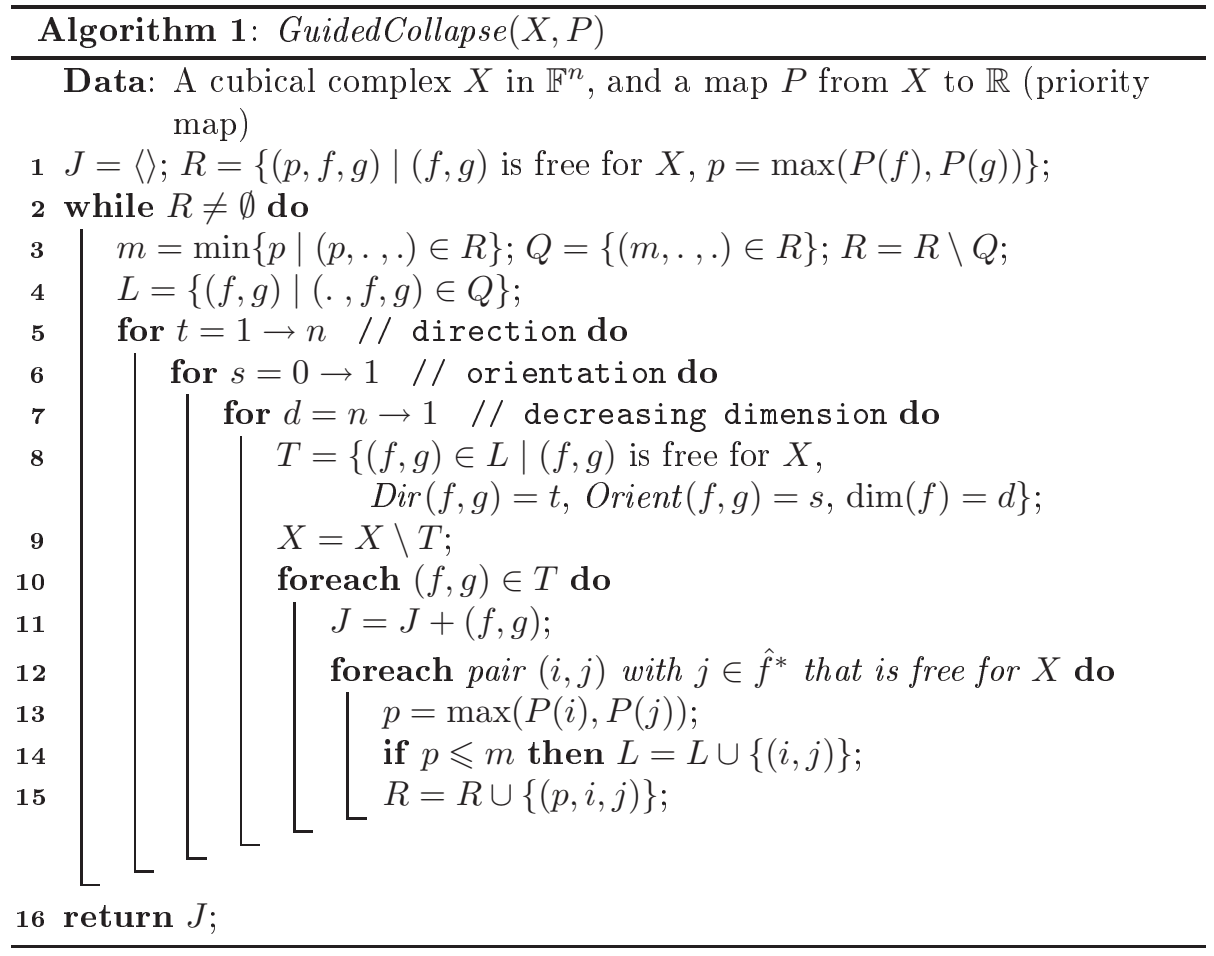

Based on Cor. 5, the following property is straightforward.

Proposition 6. Whatever the complex $X$ and the map $P$ from $X$ to $\mathbb{R}, X$ collapses onto GuidedCollapse $(X, P)$.

Algorithm GuidedCollapse may be implemented to run in $O(N \log N)$ time complexity, where $N$ denotes the cardinality of $X$, using a balanced binary tree data structure (see [16]) for representing the set $R$. For this evaluation, we consider the dimension of the complex as a constant (usually 2 or 3 ). Consequently, all local operations (such as the selection of neighboring pairs at line 12, and the test that determines whether a pair is free or not) may be done in constant time. The management of $R$ (lines $1,2,3,15)$ has a cost in $O(\log N)$ for each operation. Remark that the contents of $T$ at different iterations form disjoint sets, thus the cumulated cost of lines 8-15, during the whole execution of the algorithm, is not greater than $O(N \log N)$. The same can be said for the set $L$ and lines $4-15$.

To conclude this section, we introduce the notion of a flow graph associated to a given collapse sequence.

A (finite directed) graph is a pair $(V, E)$ where $V$ is a finite set and $E$ is a subset of $V \times V$. An element of $V$ is called a vertex, an element of $E$ is called 
an arc. A path in $(V, E)$ is a sequence $\left\langle v_{i}\right\rangle_{i=0}^{\ell}$ of vertices such that $\ell \geqslant 0$ and for all $i \in\{1, \ldots, \ell\}$, we have $\left(v_{i-1}, v_{i}\right) \in E$. The number $\ell$ is the length of the path. If $\ell=0$ the path is said trivial. If $v_{0}=v_{\ell}$ the path is a cycle. The graph is acyclic if it does not contain any non-trivial cycle.

Definition 7. Let $X$ be a complex and $J=\left\langle\left(f_{i}, g_{i}\right)\right\rangle_{i=1}^{\ell}$ be a collapse sequence from $X$. For any $k \in\{1, \ldots, \ell\}, \ell \geqslant 0$, we set $X_{k}=X \backslash\left\{f_{i}, g_{i}\right\}_{i=1}^{k}$. We set $E_{1}=\left\{\left(g_{i}, f_{i}\right)\right\}_{i=1}^{\ell}$ and $E_{2}=\bigcup_{k=1}^{\ell}\left\{\left(f_{k}, g\right) \mid g \in \hat{f}_{k}^{*} \cap X_{k}\right\}$.

The flow graph associated to $J$ is the (directed) graph whose vertex set is $X$ and whose edge set is $E=E_{1} \cup E_{2}$.

This definition is illustrated in Fig. 7. It can be easily seen that, whatever the complex $X$ and the collapse sequence $J$ from $X$, the flow graph associated to $J$ is acyclic.

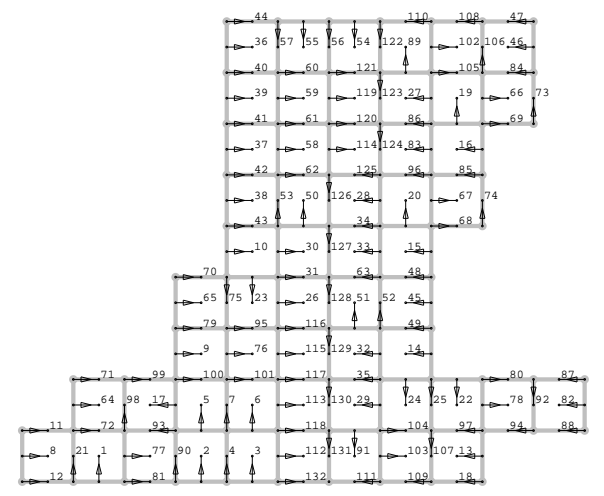

(a)

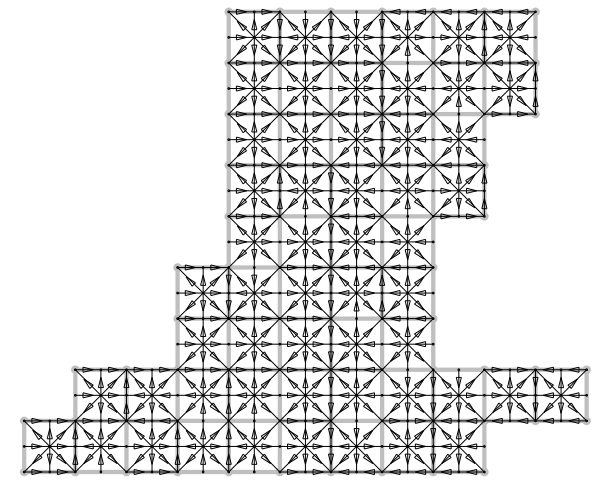

(b)

Figure 7: (a) A collapse sequence $J$. Each pair $\left(f_{i}, g_{i}\right)$ of $J$ is depicted by an arrow from $g_{i}$ to $f_{i}$. The numbers indicate the indices of the pairs in $J$. (b) The flow graph associated to $J$.

In Fig. 8 we illustrate flow graphs associated with collapse sequences that were obtained by the above algorithm using two different priority maps. For the 
sake of readability, we only display a spanning directed forest (which is a tree, in this case) extracted from the flow graph. For Fig. 8(d), the priority map is the Euclidean distance map displayed in Fig. 8(b), and for Fig. 8(e), the priority map is the projection radius map displayed in Fig. 8(c). We observe that each branch of a $\lambda$-medial axis (level set of Fig. 8(c)) corresponds, roughly speaking, to a path in the flow graph Fig. 8(e), but this is not true if we consider the flow graph Fig. 8(d).

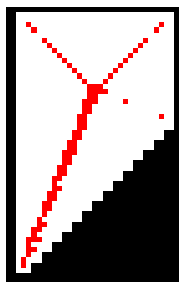

(a)

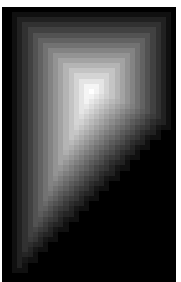

(b)

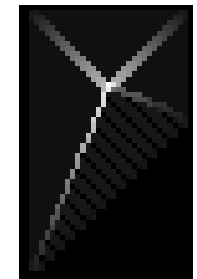

(c)

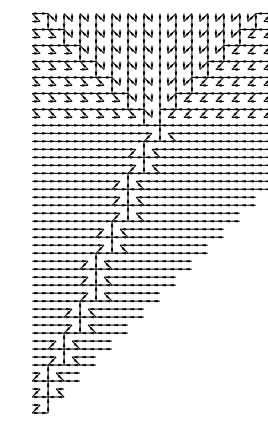

(d)

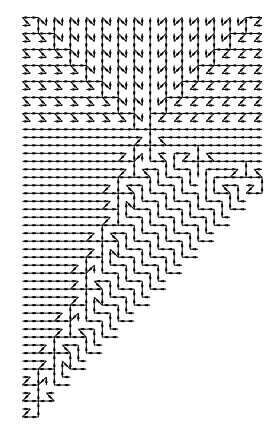

(e)

Figure 8: (a) Original object $X$ (complex). Superimposed: centers of maximal included Euclidean balls. (b) Euclidean distance map of $X$ (named $E D$ ). (c) Projection radius map of $X$ (named $P R$ ). (d) Spanning forest extracted from the flow graph associated to the sequence GuidedCollapse $(X, E D)$. (e) Spanning forest extracted from the flow graph associated to the sequence GuidedCollapse $(X, P R)$.

\section{Upstream of a vertex and its valuation}

From now, we consider a collapse sequence $J=\left\langle\left(f_{i}, g_{i}\right)\right\rangle_{i=1}^{\ell}$ from a complex $X$, and its associated flow graph $\left(X, E=E_{1} \cup E_{2}\right)$. Using the notations of Def. 7, any pair $\left(f_{k}, g_{k}\right)$ of $J$ is free for $X_{k-1}$, and we have $X=X_{0} \searrow \ldots \searrow X_{\ell}$. We define $F=\left\{f_{i}\right\}_{i=1}^{\ell}, G=\left\{g_{i}\right\}_{i=1}^{\ell}$ and $X_{J}=F \cup G$.

Let $x \in X$, we denote by $\Gamma(x)$ the set of successors of $x$ in the acyclic graph $(X, E)$, that is, $\Gamma(x)=\{y \in X \mid(x, y) \in E\}$, and we denote by $\Gamma^{-1}(x)$ the set of predecessors of $x$ in this graph, that is, $\Gamma^{-1}(x)=\{y \in X \mid(y, x) \in E\}$. We denote by $d^{+}(x)$ the outer degree of the vertex $x$ in the graph $(X, E)$, that is, the number of successors of $x$. 
We call upstream of $x$ the set of all vertices that are ancestors of $x$ in the flow graph, that is, the set $U(x)=\{y \in X \mid$ there is a path from $y$ to $x$ in $(X, E)\}$.

In a collapse sequence, certain pairs can be swapped or eliminated, yielding another collapse sequence (see e.g. Cor. 4). Intuitively, the elements of the upstream of a face $x$ of $X$ are those that must indeed be collapsed before $x$ can itself collapse. In Fig. 9 we show several examples of vertices and their upstream.
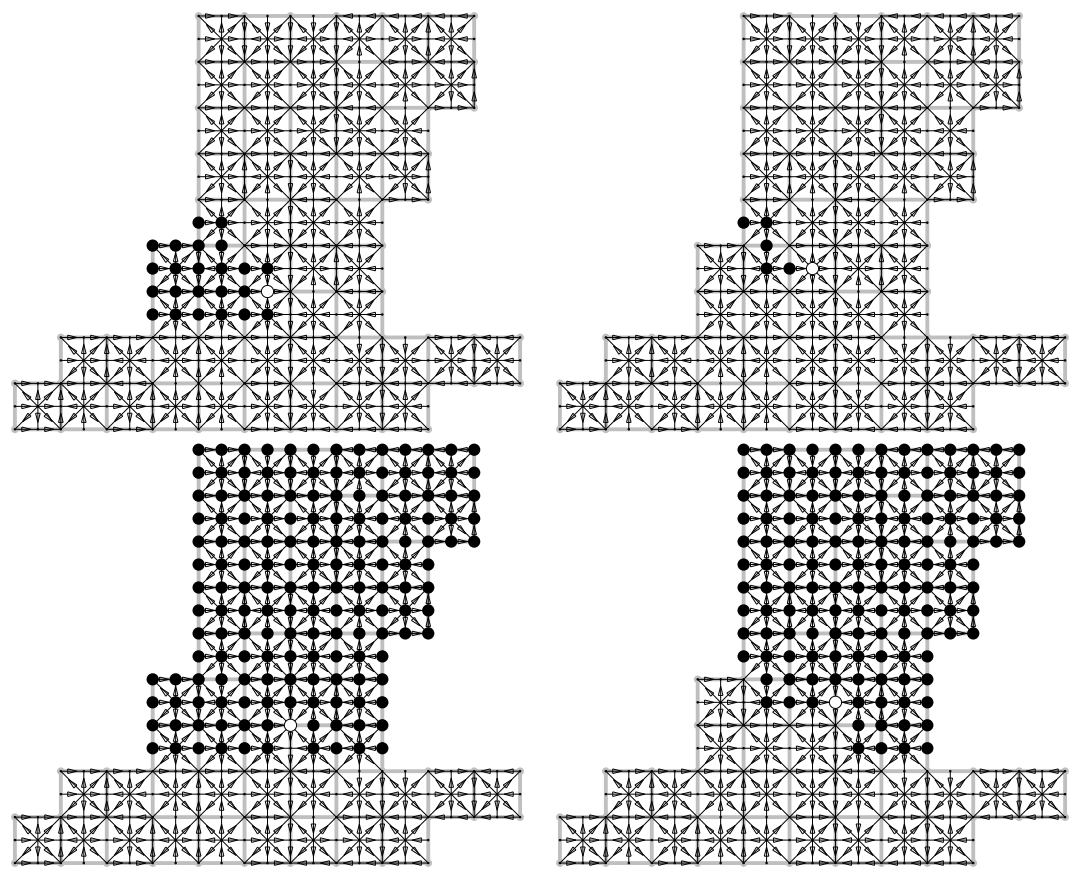

Figure 9: Four vertices (white discs) and their respective upstreams (black discs), for the same flow graph as in Fig. 7.

Let $L$ be a map from $X$ to $\mathbb{R} \cup\{+\infty\}$. Roughly speaking, the map $\widetilde{L}$ defined below cumulates, for each vertex $x$, the values of $L$ on all vertices of the upstream of $x$.

Definition 8. Let $L$ be a map from $X$ to $\mathbb{R} \cup\{+\infty\}$. We define the map $\widetilde{L}$ such that, for any $x \in X$ :

$$
\widetilde{L}(x)=L(x)+\sum_{y \in \Gamma^{-1}(x)} \widetilde{L}(y) / d^{+}(y)
$$

Notice that this definition is recursive, and that it makes sense since the graph $(X, E)$ is acyclic. Intuitively, the division by $d^{+}(y)$ is motivated by the fact that a value must not be taken in account several times in the sum. The values $\widetilde{L}(x)$ can be computed thanks to the following recursive program (algorithm 3 ). 

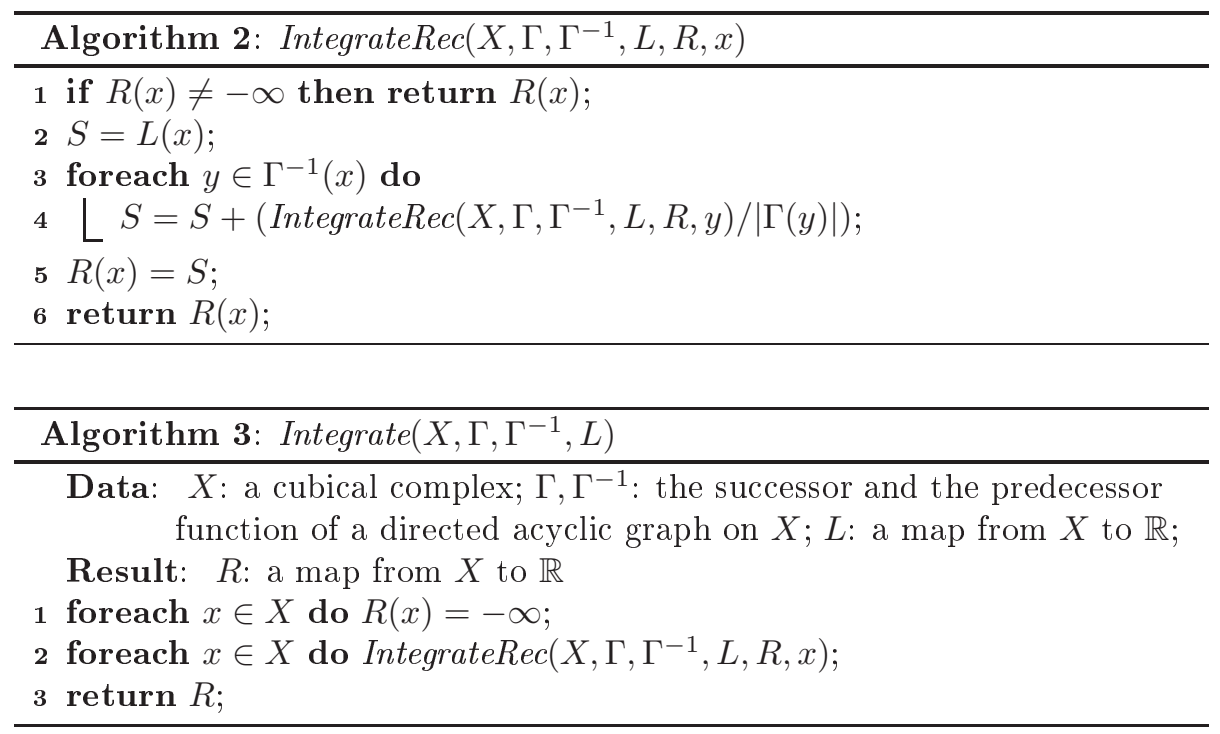

Observe that lines 1 and 5 of Alg. (2) ensure that at most $\left|\Gamma^{-1}(x)\right|$ recursive calls will occur for each element $x$, thus the overall time complexity of Alg. (3) is in $O(N+|E|)$, where $N=|X|$. Again, if we consider the dimension of the complex as a constant $(n=2$ or $n=3)$, Alg. (3) is in $O(N)$ since $|E| \leqslant 3^{n} N$.

Two particularly simple functions $L$ yield meaningful indicators associated to the elements of $X$. Let us first consider the function $L_{1}$ such that $L_{1}(x)=1$ if $\operatorname{dim}(x)=n$, and $L_{1}(x)=0$ otherwise. The map $\widetilde{L}_{1}$ associates, to each element $x$ of $X$, the "area of $U(x)$ " (or its volume in $3 \mathrm{D}$ ). Now, let us consider $L_{2}=1_{B(X)}$, where $B(X)$ is the set of all faces that are free for $X$. We call $B(X)$ the border of $X$. In other words, $L_{2}(x)=1$ if $x \in B(X)$, and $L_{2}(x)=0$ otherwise. The map $\widetilde{L}_{2}$ associates, to each element $x$ of $X$, a measure (length in $2 \mathrm{D}$, surface area in 3D) of $U(x) \cap B(X)$.

Fig. 10 $\left(a_{1}, a_{2}\right)$ show the maps $L_{1}$ and $L_{2}$ respectively, for the same object $Y$. The maps $\widetilde{L}_{1}$ and $\widetilde{L}_{2}$ are displayed in Fig. $10\left(b_{1}, b_{2}\right)$.

\section{Topological maps}

In this section, we introduce the notion of topological map. A topological map based on a collapse sequence $J$ is a map on the elements of $X$ that satisfies certain conditions relative to $J$ and its associated flow graph. Then, we prove an important property of such maps: if $M$ is a topological map, then any level set of $M$ is homotopy-equivalent to $X$. In Sec. 7, we will show how to build such a map, based on any given function on $X$.

Definition 9. Let $M$ be a map from $X$ to $\mathbb{R} \cup\{+\infty\}$. We say that $M$ is a topological map on $X$ (based on $J$ ) if: 


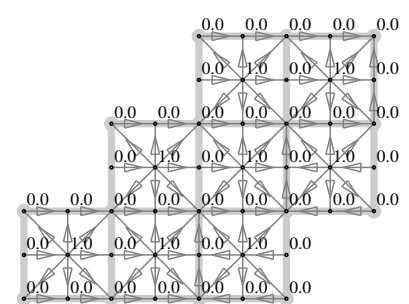

$\left(a_{1}\right)$

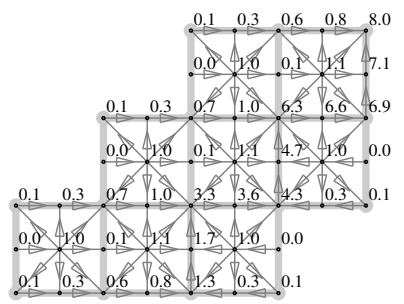

$\left(b_{1}\right)$

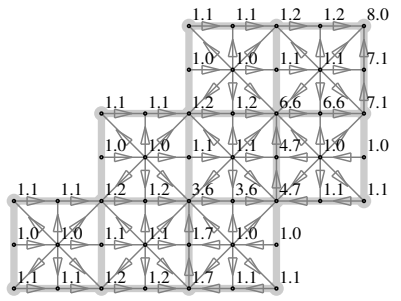

$\left(c_{1}\right)$

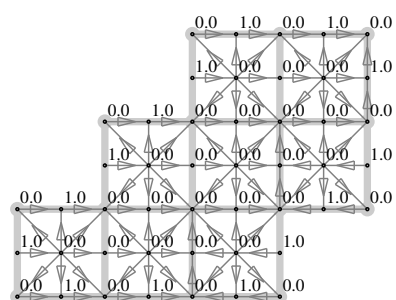

$\left(a_{2}\right)$

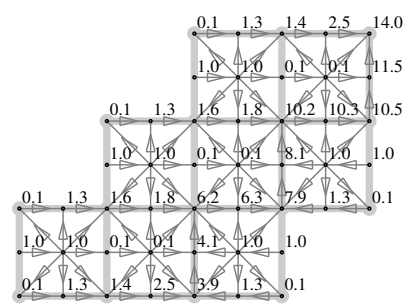

$\left(b_{2}\right)$

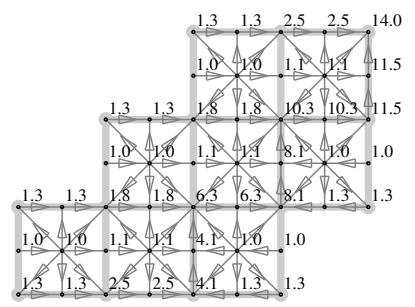

$\left(c_{2}\right)$

Figure 10: $\left(a_{1}, a_{2}\right)$ Maps $L_{1}$ and $L_{2}$ on the same complex $Y$. $\left(b_{1}, b_{2}\right)$ Maps $\widetilde{L}_{1}$ and $\widetilde{L}_{2}$. For the sake of readability, only one digit after the decimal point is displayed. $\left(c_{1}, c_{2}\right)$ Results $S_{1}$ and $S_{2}$ of the AlphaTM operator on $\widetilde{L}_{1}$ and $\widetilde{L}_{2}$, respectively, with $\alpha=0.1$.

i) for all $(g, f)$ in $E_{1}, M(g)=M(f)$; and

ii) for all $(f, g)$ in $E_{2}, M(g)>M(f)$; and

iii) for all $g$ in $X \backslash X_{J}, M(g)=+\infty$.

Let $\alpha$ be a positive real number. If we replace ii) with the stronger requirement:

ii') for all $(f, g)$ in $E_{2}, M(g) \geqslant M(f)+\alpha$,

then we say that $M$ is an $\alpha$-topological map on $X$ (based on $J$ ).

The notion of topological map is inspired from the one of discrete Morse function (see [22]). A topological map can be seen (apart from the infinite values) as a particular case of discrete Morse function, and Th. 12 could also be proved using results of [22]. However as the proof is short we include it for the sake of self-containedness. 


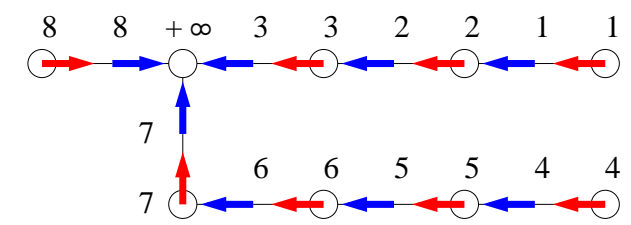

Figure 11: A 1-complex $X$, a flow graph on $X$ (black arrows for arcs of $E_{1}$, red arrows for arcs of $E_{2}$ ), and a (1-)topological map $M$ on $X$ (numbers).

Let $\lambda \in \mathbb{R} \cup\{+\infty\}$, we define $M_{\lambda}=\{x \in X \mid M(x) \geqslant \lambda\}$, the (upper) level set of $M$ at level $\lambda$. The main property of a topological map $M$ is that any level set of $M$ is homotopy-equivalent to $X$, as implied by the following theorem (Th. 12, see Fig. 12 for an illustration). The two next propositions will be used for proving it.

Proposition 10. Let $\left(g_{k}, f_{k}\right) \in E_{1}$. For all $g^{\prime}$ in $\hat{f}_{k}{ }^{*}$ such that $g^{\prime} \neq g_{k}$, we have $\left(f_{k}, g^{\prime}\right) \in E_{2}$.

Proof. We know that $\left(f_{k}, g_{k}\right)$ is free for $X_{k-1}$, implying that $X_{k-1}$ is a complex and that $f_{k} \in X_{k-1}$, hence $\hat{f}_{k} \subseteq X_{k-1}$. Since $X_{k}=X_{k-1} \backslash\left\{f_{k}, g_{k}\right\}$ and $g^{\prime} \neq g_{k}$, by definition of $E_{2}$ we have $\left(f_{k}, g^{\prime}\right) \in E_{2}$.

Proposition 11. Let $M$ be a topological map on $X$, based on $J$. Let $s=$ $\min \{M(x) \mid x \in X\}$. If $s<+\infty$, let $t=\min \{M(x) \mid x \in X$ and $M(x)>s\}$, otherwise let $t=+\infty$. Then, $X$ collapses onto $M_{t}$. Moreover, $M$ (restricted to the elements of $M_{t}$ ) is a topological map on $M_{t}$.

Proof. If $s=+\infty$ then the property trivially holds, let us assume $s<+\infty$. Let $S=\left\{\left(x_{i}, y_{i}\right)\right\}_{i=1}^{k}$ be the set of all pairs of $E_{1}$ such that $M\left(x_{i}\right)=M\left(y_{i}\right)=s$. By definition of $t$, all elements of $X \backslash M_{t}$ are in these pairs. Let $(x, y)$ be any of these pairs, and let $\left(f_{j}, g_{j}\right)$ denote the pair of $J$ such that $f_{j}=y$ and $g_{j}=x$. We know that $x \subseteq y$.

We claim that $(y, x)$ is free for $X$. To prove this, suppose that $y^{\prime}$ is a face of $X$ such that $y^{\prime} \neq y$ and $x \subseteq y^{\prime}$. As $(y, x)=\left(f_{j}, g_{j}\right)$ is free for $X_{j-1}$, we deduce that there exists a pair $\left(f_{\ell}, g_{\ell}\right)$ in $J$ such that $\ell<j$ and $f_{\ell}=y^{\prime}$. Let $x^{\prime}=g_{\ell}$. Thus $\left(x^{\prime}, y^{\prime}\right) \in E_{1}$, and by Prop. 10 we have $\left(y^{\prime}, x\right) \in E_{2}$, hence $M(x)>M\left(y^{\prime}\right)$, in contradiction with the definition of $s$ and $S$.

This proves that all pairs $\left(y_{i}, x_{i}\right)$ in $S$ are free for $X$. As all these pairs appear in the collapse sequence $J$, we know that all $y_{i}$ are distinct faces of $X$, and by Cor. 2 we conclude that $X$ collapses onto $M_{t}$.

The fact that $M$ is a topological map on $M_{t}$ is a consequence of Cor. 4 .

Th. 12 is a straightforward consequence of Prop. 11.

Theorem 12. Let $M$ be a topological map on $X$. Whatever the number $\lambda \in$ $\mathbb{R} \cup\{+\infty\}$, the complex $X$ collapses onto $M_{\lambda}$. 


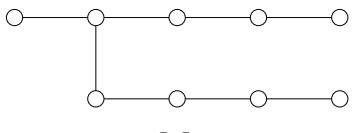

$M_{1}$

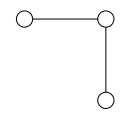

$M_{7}$

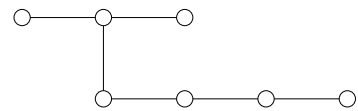

$M_{3}$

$M_{9}$

Figure 12: Level sets of the topological map $M$ of Fig. 11 at levels 1, 3, 7 and 9.

The next theorem (Th. 14) expresses the stability of our skeletonization scheme, with respect to the variations of the filtering parameter.

Let $S, T$ be two subsets of $\mathbb{R}^{n}$. We set

$$
H(S \mid T)=\max _{s \in S}\left\{\min _{t \in T}\{d(s, t)\}\right\},
$$

and $d_{H}(S, T)=\max \{H(S \mid T), H(T \mid S)\}$ is the Hausdorff distance between $S$ and $T$.

Let $X$ be a complex in $\mathbb{F}^{n}$, we denote by $\mathcal{S}(X)$ the union of all faces of $X$, called the support of $X$. For comparing two complexes $X$ and $Y$, we consider the Hausdorff distance between their supports $\mathcal{S}(X)$ and $\mathcal{S}(Y)$.

The following property follows easily from the definitions.

Proposition 13. Let $Y$ be a complex, let $S$ be a set of pairs that are free for $Y$, and let $Z$ be the set of all faces that are in the pairs of $Y$. Then, $d_{H}(\mathcal{S}(Y), \mathcal{S}(Y \backslash$ $Z)) \leqslant 1$.

The proof of Th. 14 is quite similar to the one of Prop. 11.

Theorem 14. Let $\alpha, \lambda \in \mathbb{R}, \alpha>0, \lambda \geqslant 0$. Let $k \in \mathbb{N}$. Let $M$ be an $\alpha$ topological map on $X$. Then, $d_{H}\left(\mathcal{S}\left(M_{\lambda}\right), \mathcal{S}\left(M_{\lambda+k \alpha}\right)\right) \leqslant k$.

Proof. Clearly if the property holds for $k=1$, it also holds for any $k$. We assume now that $k=1$. If $\lambda=+\infty$ then the property trivially holds, let us assume $\lambda<+\infty$. Let $S=\left\{\left(x_{i}, y_{i}\right)\right\}_{i=1}^{k}$ be the set of all pairs of $E_{1}$ such that $\lambda \leqslant M\left(x_{i}\right)=M\left(y_{i}\right)<\lambda+\alpha$. Let $(x, y)$ be any of these pairs, and let $\left(f_{j}, g_{j}\right)$ denote the pair of $J$ such that $f_{j}=y$ and $g_{j}=x$. We know that $x \subseteq y$.

Suppose that $y^{\prime}$ is a face of $M_{\lambda}$ such that $y^{\prime} \neq y$ and $x \subseteq y^{\prime}$. As $(y, x)=$ $\left(f_{j}, g_{j}\right)$ is free for $X_{j-1}$, we deduce that there exists a pair $\left(f_{\ell}, g_{\ell}\right)$ in $J$ such that $\ell<j$ and $f_{\ell}=y^{\prime}$. Let $x^{\prime}=g_{\ell}$. Thus $\left(x^{\prime}, y^{\prime}\right) \in E_{1}$, and by Prop. 10 we have $\left(y^{\prime}, x\right) \in E_{2}$, thus $M(x)>M\left(y^{\prime}\right)+\alpha$, hence $M\left(y^{\prime}\right)<M(x)-\alpha<\lambda$, in contradiction with the fact that $y^{\prime}$ belongs to $M_{\lambda}$.

This proves that all pairs $\left(y_{i}, x_{i}\right)$ in $S$ are free for $M_{\lambda}$, and by Prop. 13, we deduce the result. 


\section{Topological map induced by an arbitrary map}

In this section, we show that given any map $L$ on $X$, we can define and compute a topological map that is "close to" $L$, more precisely it is the lowest map above $L$ that is a topological map.

Definition 15. Let $L$ be any map from $X$ to $\mathbb{R} \cup\{+\infty\}$, and let $\alpha$ be a positive real number. We consider a map $M$ such that:

a) $M$ is an $\alpha$-topological map; and

b) for all $f$ in $X_{J}, M(f) \geqslant L(f)$; and

c) $M$ is minimal for conditions a) and b), that is, any map $M^{\prime}$ verifying both

a) and b) is such that $M^{\prime} \geqslant M$.

As stated by the following property, $M$ is uniquely defined. We say that the map $M$ is the $\alpha$-topological map induced by $L$.

Proposition 16. Let $M$ and $M^{\prime}$ be two maps that verify conditions a), b) and c) of Def. 15. Then, we have $M=M^{\prime}$.

Proof. Since for any couple $(x, y)$ of $E_{1}$ we have $M(x)=M(y)$ and $M^{\prime}(x)=$ $M^{\prime}(y)$, we observe that either $M$ and $M^{\prime}$ are equal, or they differ on a certain number of couples of $E_{1}$. Suppose that $(x, y)$ is a couple of $E_{1}$ such that $M^{\prime}(x) \neq$ $M(x)$. Without loss of generality, we assume that (1) $M^{\prime}(x)>M(x)$ (hence also $M^{\prime}(y)>M(y)$ ), and (2) no face $z$ of $X$ verifies both $M^{\prime}(z) \neq M(z)$ and $M^{\prime}(z)<M^{\prime}(x)$.

Consider the map $M^{\prime \prime}$ such that $M^{\prime \prime}(x)=M^{\prime \prime}(y)=M(x)=M(y)$, and $\forall z \in X \backslash\{x, y\}, M^{\prime \prime}(z)=M^{\prime}(z)$. Obviously $M^{\prime \prime}$ verifies condition b) above. We claim that $M^{\prime \prime}$ is an $\alpha$-topological map, contradicting the minimality of $M^{\prime}$; proving this claim will achieve the proof.

Conditions i) and iii) of Def. 9 are easily verified. Condition ii) must only be checked for arcs adjacent to $x$ and $y$, since for all other arcs, $M^{\prime \prime}$ and $M^{\prime}$ coincide.

If $(z, x)$ is an arc of $E$ then necessarily $(z, x) \in E_{2}$. We have $M^{\prime}(x) \geqslant$ $M^{\prime}(z)+\alpha$, hence $M^{\prime}(z)<M^{\prime}(x)$ and by $(2), M^{\prime}(z)=M(z)$. We also have $M(x) \geqslant M(z)+\alpha$, and by definition of $M^{\prime \prime}$ we deduce $M^{\prime \prime}(x) \geqslant M^{\prime \prime}(z)+\alpha$.

If $(y, z)$ is an $\operatorname{arc}$ of $E$ then necessarily $(y, z) \in E_{2}$. We have $M^{\prime}(z) \geqslant M^{\prime}(y)+$ $\alpha$. By definition of $M^{\prime \prime}$, we know that $M^{\prime}(z)=M^{\prime \prime}(z)$ and $M(y)=M^{\prime \prime}(y)$; and by $(1), M^{\prime}(y)>M^{\prime \prime}(y)$. From all this we conclude that $M^{\prime \prime}(z) \geqslant M^{\prime \prime}(y)+\alpha$.

This notion is illustrated in Fig. 13. Below, we give an algorithm that computes the $\alpha$-topological map induced by any given map on $X$. Before this, let us recall briefly the notions of rank and topological sort (an introduction to topological sort, including definition, properties and algorithm, can be found, e.g., in [16]). Let $G=(V, E)$ be an acyclic graph and let $x \in V$, the rank of $x$ in $G$ is the length of the longest path in $G$ that ends in $x$. The topological sort of $G$ is an operation that results in a partition $\left\{V^{r}\right\}_{r=0}^{r=k}$ of $V$ such that each $V^{r}$ is the subset of $V$ containing all vertices of rank $r$. 


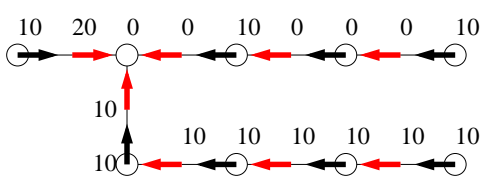

(a)

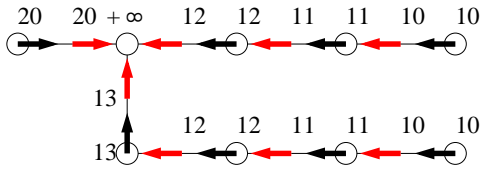

(b)

Figure 13: (a) A map $L$ on the complex $X$ of Fig. 11. (b) The 1-topological map induced by $L$.

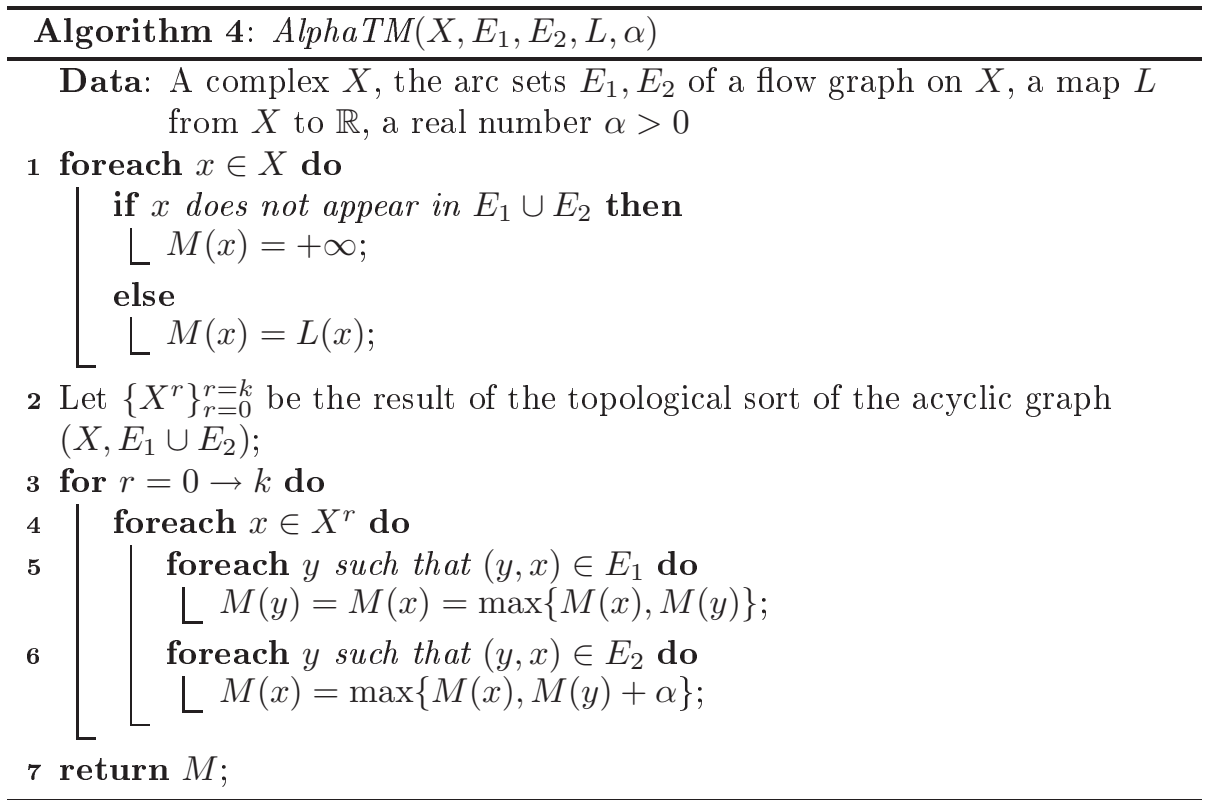

Proposition 17. Let $M$ be a map from $X$ to $\mathbb{R}$, and let $\alpha$ be real number, $\alpha>0$. The result of AlphaTM $\left(X, E_{1}, E_{2}, M, \alpha\right)$ is the $\alpha$-topological map induced by $M$.

Proof. Condition iii) of Def. 9 is ensured by line 1. In lines 3-6, each vertex of the flow graph is examined exactly once, and, due to the order of scanning (lines 3,4 ) and by definition of topological sort, the final values $M(y)$ of all predecessors $y$ of the current vertex $x$ have been computed before it is examined. For vertices that have no predecessor, the output value of $M$ is equal to the input value. Otherwise, lines 5 and 6 ensure that conditions i) and ii') of Def. 9 hold. By construction, the minimality of $M$ is guaranteed.

Let $N=|X|$ and $M=\left|E_{1} \cup E_{2}\right|$. The time complexity of the topological sort is in $O(N+M)$ (see [16]). Since the sets $X^{r}$ form a partition of $X$, the overall time complexity of Alg. (4) is also in $O(N+M)$, and in $O(N)$ if the dimension of the complex is considered as a constant $(n=2$ or $n=3)$. 


\section{Computing hierarchic skeletons}

Let us now summarize our method to produce families of filtered homotopic skeletons (see algorithm 5). It is assumed here that $X$ is a pure $n$-complex in $\mathbb{F}^{n}$, that is, a complex in which each face is included in an $n$-face.

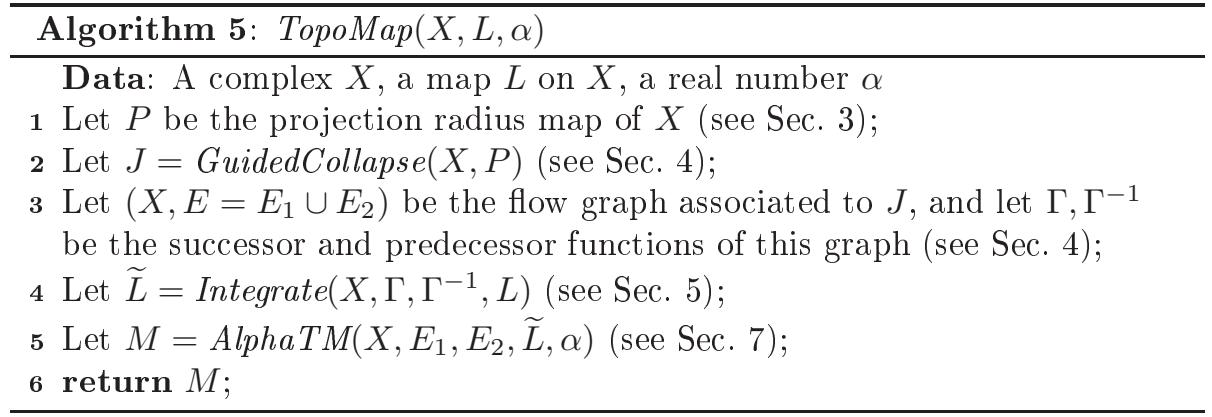

First, we compute the projection radius map (Sec. 3) on the $n$-faces of $X$, and extend it to the other elements of $X$ (if $y \in X$ is not an $n$-face, then we set $P(y)$ to the max of $P\left(x_{i}\right)$ where the $x_{i}$ 's are all $n$-faces that include $y$ ).

Using algorithm 1 (Sec. 4) we build a collapse sequence and a flow graph on $X$.

By construction, the upstream (Sec. 5) of any vertex $x$ of this flow graph is composed by elements of $X$ that, in any family of filtered skeletons, should disappear before $x$ does.

Integrating information given by map $L$ (Sec. 5) allows us to associate, to each element $x$ of $X$, a value $\widetilde{L}(x)$ that represents a measure of the upstream of $x$. The lower this value, the sooner the point $x$ may disappear.

Then, thanks to algorithm 4 (Sec. 7), we produce a topological map $M$ based on this measure. Thanks to Th. 12, we know that any level set of $M$ is homotopy-equivalent to $X$. Therefore, filtered (i.e., pruned) skeletons are obtained by thresholding the map $M$; lowest levels of threshold correspond to highest levels of detail. Some results are shown in Fig. 14 and Fig. 15, using map $L_{2}$.

Another interesting map is $L_{3}$, which associates to each point $x$ of $X$ the bisector angle of $x$, that is, the maximal angle $\widehat{a x b}$ with $a, b$ any two points in the extended projection of $x$ on $\bar{X}$ (see [18]). In the next section, we will see that $L_{3}$ yields particularly good results.

According to the time complexity evaluation of previous algorithms, the overall time complexity of Alg. (5) is in $O(N \log N)$, where $N=|X|$.

\section{Quality assessment and comparisons}

In order to assess the quality of the produced skeletons, and to compare them to those obtained by other methods, we use stability w.r.t. rotations as our quality criterion, following a methodology introduced in [13]. 

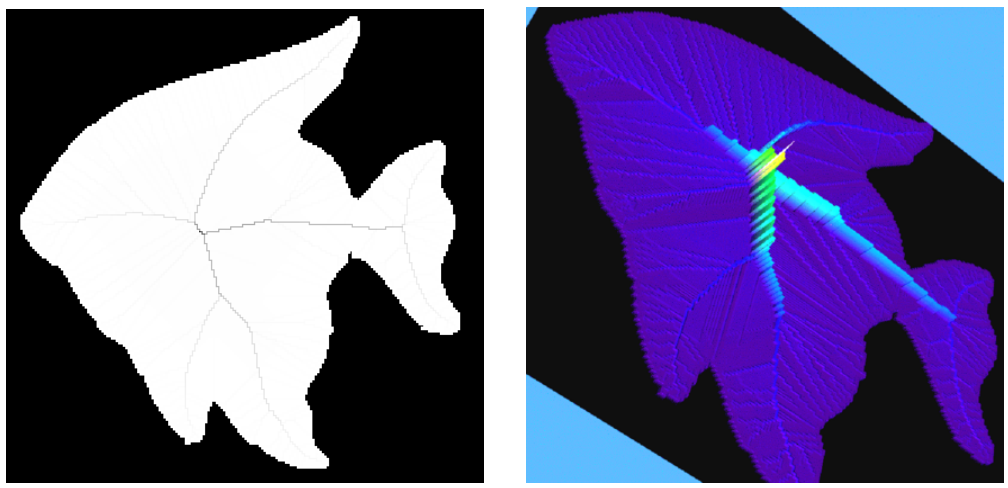

Figure 14: Two renderings of the result of the TopoMap operator, on the same object $X$ as in Fig. 6 and the map $L_{2}$.

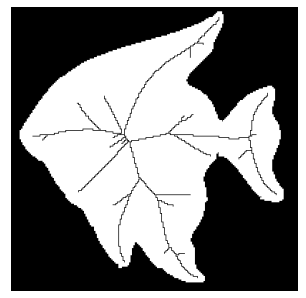

(a)

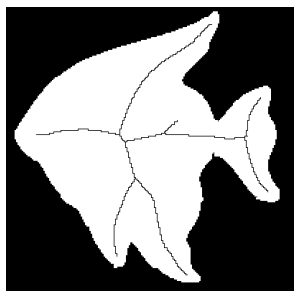

(b)

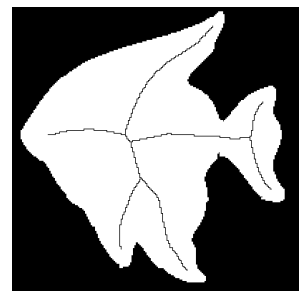

(c)

Figure 15: (a,b,c) Three level sets of $\operatorname{TopoMap}\left(X, L_{2}\right)$, at values 25, 48 and 72 , respectively.

Rotation invariance is an important property of skeleton that holds in the continuous framework. If $R_{\theta}$ denotes the rotation of angle $\theta$ and center 0 , and $S$ denotes the skeleton transform, the rotation invariance property states that $S\left(R_{\theta}(X)\right)=R_{\theta}(S(X))$, whatever $X$ and $\theta$.

In a discrete framework, this property can only hold for particular cases (e.g., when $\theta$ is a multiple of 90 degrees). Nevertheless, we can experimentally measure the dissimilarity between $S\left(R_{\theta}(X)\right)$ and $R_{\theta}(S(X))$ for different instances of $X$ and $\theta$, and different definitions of skeleton. The lower this dissimilarity, the more stable under rotation the method is.

Let us now describe more precisely the methodology that we used for this experimental evaluation.

Let $X$ be a finite subset of $\mathbb{Z}^{n}$. Notice that a cubical complex has a natural embedding in $\mathbb{Z}^{n}$ and can thus be treated as a point set. Let $Y \subseteq X$, we set $\operatorname{RDT}_{X}(Y)=\bigcup_{y \in Y} B_{d(y, \bar{X})}^{<}(y)$, where $B_{r}^{<}(x)=\left\{y \in \mathbb{Z}^{n} \quad \mid d(x, y)<r\right\}$. The transformation $R D T_{X}$ is sometimes called reverse distance transform [15].

It is well known that any object can be fully reconstructed from its medial axis, more precisely, we have $X=R D T_{X}(Y)$ whenever $Y$ is the the set of all 
centers of maximal balls of $X$. However, this is no longer true if we consider filtered skeletons.

Then, it is interesting to measure how much information about the original object is lost when we raise the filtering parameter. Considering a skeletonization procedure $S_{\lambda}$ where $\lambda$ is the only parameter, we set

$$
\rho_{X}(\lambda)=\frac{\left|X \backslash R D T_{X}\left(S_{\lambda}(X)\right)\right|}{|X|} .
$$

In words, $\rho_{X}(\lambda)$ is the area of the difference between $X$ and the set reconstructed from its filtered skeleton, divided by the area of $X$. We call $\rho_{X}(\lambda)$ the (normalized) residual (of $X$, corresponding to filtering value $\lambda$ ).

Obviously, for different skeletonization methods the filtering parameter does not play the same role. To ensure a fair evaluation we will compare the results of different methods for approximately equal values of their residuals, rather than for equal values of their parameters.

For comparing shapes or skeletons, we use the Hausdorff distance (see Sec. 6), and also a dissimilarity measure proposed by M.P. Dubuisson and A.K. Jain [20] as an alternative to the Hausdoff distance. The drawback of Hausdorff distance for measuring shape dissimilarity is its extreme sensibility to outliers, the latter measure avoids this drawback.

Let $X, Y$ be two subsets of $\mathbb{R}^{n}$. We set

$$
D(X \mid Y)=\frac{1}{|X|} \sum_{x \in X} \min _{y \in Y}\{d(x, y)\},
$$

and $d_{D}(X, Y)=\max \{D(X \mid Y), D(Y \mid X)\}$ is the Dubuisson and Jain's dissimilarity measure between $X$ and $Y$ (called dissimilarity in the sequel for the sake of brevity).

We conducted our experiments on a database of 216 shapes provided by B.B. Kimia [34]. The 216 images are divided into 18 classes (birds, cars, etc.), Fig. 16 shows one (reduced) image of each class.

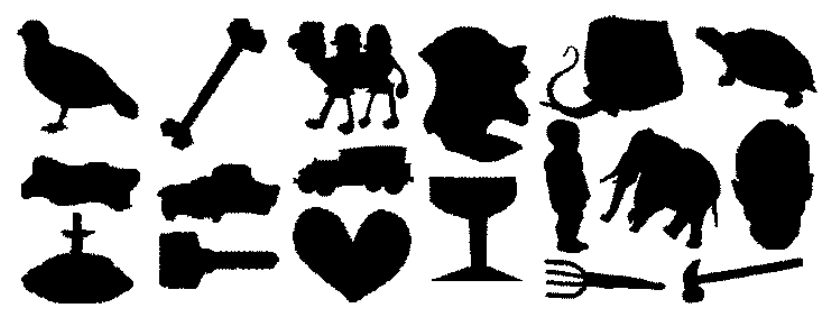

Figure 16: A sample of the 216 shapes of Kimia's database.

In those experiments, we compare three variants of our method, and two other methods which are, to the best of our knowledge, among the best ones in regard to the stability criterion which is our main concern in this work. More 
precisely, we compare:

a) Our method, using the map $L_{1}$ as described in section 5 (area indicator);

b) Our method, using the map $L_{2}$ as described in section 5 (border indicator);

c) Our method, using the map $L_{3}$ as described in section 5 (bisector angle);

d) A homotopic thinning procedure by iterative simple point removal, guided by a priority function which is the PR map, and constrained to preserve the points belonging to the $\lambda$-medial axis;

e) The skeletonization method proposed by R. Ogniewicz, using the implementation that can be found in the Stony Brook Algorithm Repository ${ }^{3}$.

\begin{tabular}{|c|c|c|c|c|c|c|c|}
\hline & $1 \%$ & $2 \%$ & $3 \%$ & $4 \%$ & $5 \%$ & $6 \%$ & all \\
\hline $\mathrm{a}$ & $\mathbf{1 . 2 2}$ & 1.58 & 1.69 & 1.63 & 1.53 & 1.46 & 1.52 \\
\hline $\mathrm{b}$ & 1.67 & 1.87 & 1.48 & 1.23 & 1.24 & 1.29 & 1.46 \\
\hline $\mathrm{c}$ & 1.56 & 1.69 & 1.44 & 1.20 & 1.10 & 1.09 & $\mathbf{1 . 3 5}$ \\
\hline $\mathrm{d}$ & 2.23 & $\mathbf{1 . 5 7}$ & $\mathbf{1 . 3 2}$ & 1.24 & 1.28 & 1.34 & 1.50 \\
\hline $\mathrm{e}$ & 1.78 & 1.78 & 1.45 & $\mathbf{1 . 1 6}$ & $\mathbf{1 . 0 9}$ & $\mathbf{1 . 0 5}$ & 1.39 \\
\hline
\end{tabular}

Table 1: Dissimilarity

\begin{tabular}{|c|c|c|c|c|c|c|c|}
\hline & $1 \%$ & $2 \%$ & $3 \%$ & $4 \%$ & $5 \%$ & $6 \%$ & all \\
\hline a & $\mathbf{7 . 8 0}$ & $\mathbf{1 1 . 7}$ & 13.7 & 14.4 & 14.0 & 13.4 & 12.5 \\
\hline b & 14.0 & 17.6 & 14.7 & 11.0 & 9.83 & 9.83 & 12.8 \\
\hline c & 12.6 & 15.4 & 14.0 & 10.9 & 8.59 & 7.99 & 11.6 \\
\hline d & 16.6 & 15.9 & 13.7 & 12.8 & 12.7 & 12.6 & 14.1 \\
\hline e & 14.6 & 15.1 & $\mathbf{1 3 . 2}$ & $\mathbf{9 . 4 8}$ & $\mathbf{7 . 9 5}$ & $\mathbf{7 . 1 9}$ & $\mathbf{1 1 . 3}$ \\
\hline
\end{tabular}

Table 2: Hausdorff distance

Tables 1 and 2 show respectively average dissimilarity and Hausdorff distance between $S\left(R_{\theta}().\right)$ and $R_{\theta}(S()$.$) , on all shapes of the database, for angles \theta$ varying between 0 and 89 degrees by steps of one degree, and for applications of the five methods yielding residuals varying between $1 \%$ and $6 \%$. Best results (lowest values) are highlighted in boldface, worst ones in italic. Fig. 17 illustrate the level of filtering obtained on a shape for these residual values.

It can be observed that variant (a) of our method performs very well with moderate filtering, while for higher residual amounts Ogniewicz' method (e) is better. In the average, variant (c) of our method performs as well as (e) on this test set.

\footnotetext{
${ }^{3}$ http://www.cs.sunysb.edu/ algorith/implement/skeleton/implement.shtml
} 


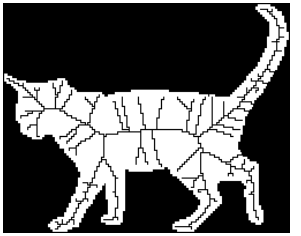

$1 \%$

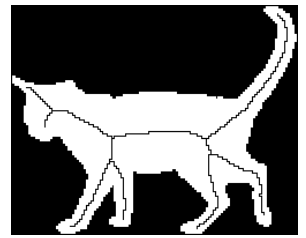

$4 \%$

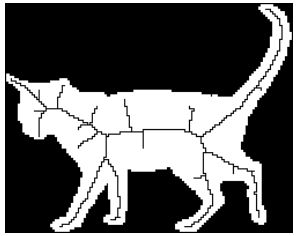

$2 \%$

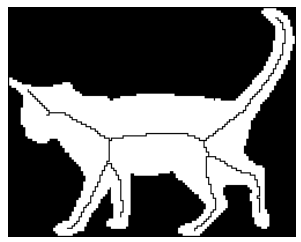

$5 \%$

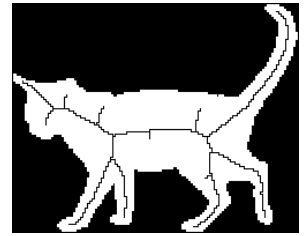

$3 \%$

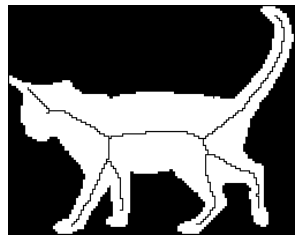

$6 \%$

Figure 17: Filtered skeletons (method b) yielding $1 \%$ to $6 \%$ residuals.

\section{The $3 \mathrm{D}$ case}

Unlike former approaches to define and compute hierarchic or multiscale skeletons, our method also applies to 3D objects for computing curvilinear skeletons. All definitions and algorithms that we gave previously are indeed valid whatever the dimension of the complex. We illustrate this by showing a few results in 3D: see Fig. 18 and Fig. 19.

Fig. 18 shows an original 3D shape $X$ (a), and the topological map $M$ of $X$ induced by $L_{2}(X)$ (b). In this $2 \mathrm{D}$ projection, the map $M$ governs the transparency. Then, (c) and (d) show two examples of filtered skeletons of $X$ obtained by thresholding $M$ at levels 100 and 1200, respectively.

Fig. 19 illustrates the effectiveness of our method in the presence of contour noise. In (a), (b) and (c), we have three versions of a 3D object with various amounts of random noise (obtained by randomly adding or suppressing simple points around the border, 1000 points for (b), 2000 points for (c)), and their respective filtered skeletons, using the same parameter value. Notice that, even if the skeletons of the noisy objects are a bit sinuous (which is not abnormal, since they are medial representations of shaky objects), they are free of spurious branches.

\section{Conclusion}

The method that we propose is guaranteed to preserve topology and is stable with respect to variations of the filtering parameter, as stated by Th. 12 and Th. 14 respectively. We compared it (in 2D) with the method of [29] and with a topology-preserving method directly based on the DLMA, regarding the stability w.r.t. rotations. This comparison is in favour of our method, as reported in Sec. 9. We also illustrate in Sec. 10 its application in 3D for obtaining curvilinear 


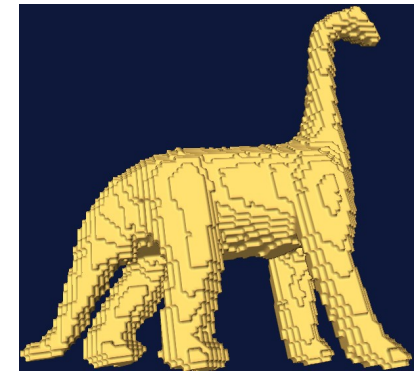

(a)

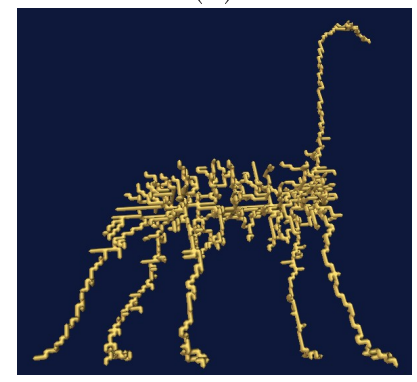

(c)

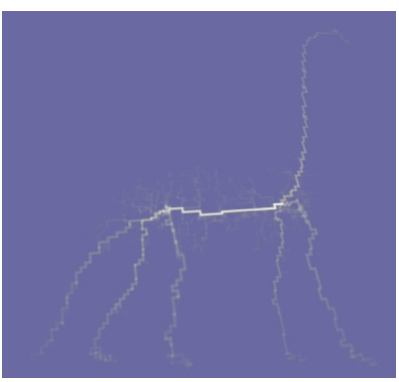

(b)

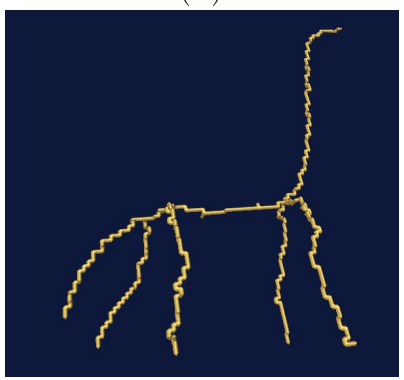

(d)

Figure 18: (a) Original shape $X$. (b) Topological map $M$ induced by $L_{2}(X)$. (c) The level set $M_{100}$, a skeleton of $X$. (d) The level set $M_{1200}$, another skeleton of $X$.

skeletons. Furthermore, our method is highly flexible: many variants can be imagined, in particular by choosing alternative valuations of the upstream. In further works, we will investigate the case of 3D surface skeletons, and the possibility to obtain skeletons only composed of voxels.

\section{References}

[1] D. Attali, J.D. Boissonnat, and H. Edelsbrunner. Stability and computation of the medial axis - a state-of-the-art report. In T. Möller, B. Hamann, and B. Russell, editors, Mathematical Foundations of Scientific Visualization, Computer Graphics, and Massive Data Exploration, pages 1-19. SpringerVerlag, 2009. to appear.

[2] D. Attali and J.O. Lachaud. Delaunay conforming iso-surface, skeleton extraction and noise removal. Computational Geometry: Theory and Applications, 19:175-189, 2001.

[3] D. Attali and A. Montanvert. Modelling noise for a better simplification of skeletons. In Procs. International Conference on Image Processing (ICIP), volume 3, pages 13-16, 1996. 


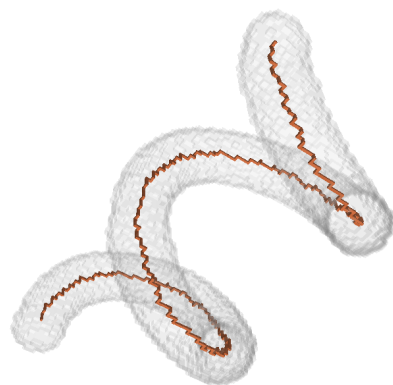

(a)

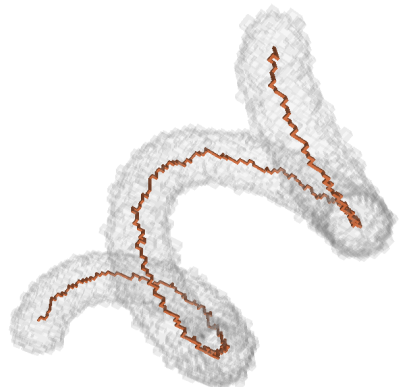

(b)

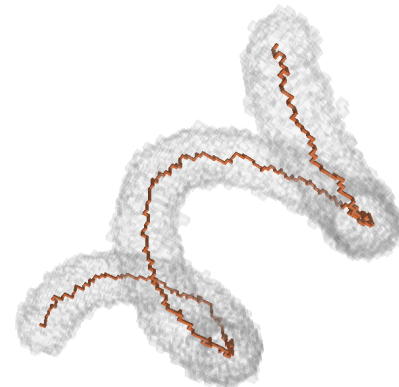

(c)

Figure 19: (a) Original shape $X$ and its filtered skeleton obtained by thresholding its topological map induced by $L_{2}(X)$ at level 1000. (b) Same operation, after addition of random noise to the contour of $X$. (c) Same operation, after addition of a stronger random noise to the contour of $X$.

[4] D. Attali, G. Sanniti di Baja, and E. Thiel. Pruning discrete and semicontinuous skeletons. In Procs. Conf. Image Analysis and Processing, volume 974 of Lecture Notes in Computer Science, pages 488-493. Springer, 1995.

[5] X. Bai, L.J. Latecki, and W.Y. Liu. Skeleton pruning by contour partitioning with discrete curve evolution. Transactions on Pattern Analysis and Machine Intelligence, 29(3):449-462, 2007.

[6] G. Bertrand and M. Couprie. A new 3D parallel thinning scheme based on critical kernels. In Discrete Geometry for Computer Imagery, volume 4245 of Lecture Notes in Computer Science, pages 580-591. Springer, 2006.

[7] G. Bertrand and M. Couprie. Two-dimensional parallel thinning algorithms based on critical kernels. Journal of Mathematical Imaging and Vision, $31(1): 35-56,2008$.

[8] S. Biasotti, L. De Floriani, B. Falcidieno, P. Frosini, D. Giorgi, C. Landi, L. Papaleo, and M. Spagnuolo. Describing shapes by geometricaltopological properties of real functions. ACM Comput. Surv., 40(4):12:112:87, October 2008.

[9] Silvia Biasotti, Dominique Attali, Jean-Daniel Boissonnat, Herbert Edelsbrunner, Gershon Elber, Michela Mortara, Gabriella Sanniti Baja, Michela Spagnuolo, Mirela Tanase, and Remco Veltkamp. Skeletal structures. In Leila Floriani and Michela Spagnuolo, editors, Shape Analysis and Structuring, Mathematics and Visualization, pages 145-183. Springer, 2008.

[10] H. Blum. A transformation for extracting new descriptors of shape. In W Wathendunn, editor, Models for the Perception of Speech and Visual Form, pages 362-380. MIT Press, 1967. 
[11] G. Borgefors, I. Ragnemalm, and G. Sanniti di Baja. The Euclidean distance transform: finding the local maxima and reconstructing the shape. In Procs. of the 7th Scandinavian Conference on Image Analysis, volume 2, pages 974-981, 1991.

[12] John Chaussard and Michel Couprie. Surface thinning in 3D cubical complexes. In International Workshop on Combinatorial Image Analysis, volume 5852 of Lecture Notes in Computer Science, pages 135-148. Springer, 2009 .

[13] John Chaussard, Michel Couprie, and Hugues Talbot. Robust skeletonization using the discrete lambda-medial axis. Pattern Recognition Letters, 32(9):1384-1394, 2011.

[14] F. Chazal and A. Lieutier. The lambda medial axis. Graphical Models, 67(4):304-331, 2005.

[15] D. Coeurjolly. d-dimensional reverse Euclidean distance transformation and Euclidean medial axis extraction in optimal time. In Discrete Geometry for Computer Imagery, volume 2886 of Lecture Notes in Computer Science, pages 327-337. Springer, 2003.

[16] T.H. Cormen, C. Leiserson, and R. Rivest. Introduction to algorithms. MIT Press, 1990.

[17] Nicu D. Cornea and Deborah Silver. Curve-skeleton properties, applications, and algorithms. 13:530-548, 2007.

[18] M. Couprie, D. Coeurjolly, and R. Zrour. Discrete bisector function and Euclidean skeleton in 2D and 3D. Image and Vision Computing, 25(10):15431556, 2007.

[19] E.R. Davies and A.P.N. Plummer. Thinning algorithms: a critique and a new methodology. Pattern Recognition, 14:53-63, 1981.

[20] M.P. Dubuisson and A.K. Jain. A modified Hausdorff distance for object matching. In Proceedings of the 12th IAPR International Conference on Pattern Recognition, volume 1, pages 566-568, 1994.

[21] A.X. Falcao, L. da Fontoura Costa, and B.S. da Cunha. Multiscale skeletons by image foresting transform and its application to neuromorphometry. Pattern Recognition, 35(7):1571-1582, 2002.

[22] R. Forman. Morse theory for cell complexes. Advances in mathematics, 134(1):90-145, 1998.

[23] Y. Ge and J.M. Fitzpatrick. On the generation of skeletons from discrete Euclidean distance maps. IEEE Transactions on Pattern Analysis and Machine Intelligence, 18(11):1055-1066, 1996. 
[24] W.H. Hesselink and J.B.T.M. Roerdink. Euclidean skeletons of digital image and volume data in linear time by the integer medial axis transform. IEEE Transactions on Pattern Analysis and Machine Intelligence, 30(12):2204-2217, 2008.

[25] A. Lieutier. Any open bounded subset of $R^{n}$ has the same homotopy type as its medial axis. In Proc. 8th ACM Symposium on Solid Modeling Applications, pages 65-75. Academic Press, 2003.

[26] L. Liu, E. Chambers, D. Letscher, and T. Ju. A simple and robust thinning algorithm on cell complexes. In Proceedings of Pacific Graphics 2010, Computer Graphics Forum, pages 1-8, 2010. to appear.

[27] G. Malandain and S. Fernández-Vidal. Euclidean skeletons. Image and Vision Computing, 16:317-327, 1998.

[28] G. Matheron. Examples of topological properties of skeletons, volume 2, pages 217-238. Academic Press, 1988.

[29] R.L. Ogniewicz and O. Kübler. Hierarchic Voronoi skeletons. Pattern Recognition, 28(33):343-359, 1995.

[30] M. Pierrot-Deseilligny, G. Stamon, and C.Y. Suen. Veinerization: a new shape description for flexible skeletonization. IEEE Transactions on Pattern Analysis and Machine Intelligence, 20(5):505-521, 1998.

[31] C. Pudney. Distance-ordered homotopic thinning: a skeletonization algorithm for 3D digital images. Computer Vision and Image Understanding, 72(3):404-413, 1998.

[32] E. Rémy and E. Thiel. Exact medial axis with Euclidean distance. Image and Vision Computing, 23(2):167-175, 2005.

[33] J. Serra. Image analysis and mathematical morphology. Academic Press, 1982.

[34] D. Sharvit, J. Chan, H. Tek, and B.B. Kimia. Symmetry-based indexing of image databases. Journal of Visual Communication and Image Representation, 9(4):366-380, 1998.

[35] K. Siddiqi, S. Bouix, A. Tannenbaum, and S. Zucker. The Hamilton-Jacobi skeleton. In International Conference on Computer Vision (ICCV), pages 828-834, 1999.

[36] Kaleem Siddiqi and Stephen Pizer. Medial Representations: Mathematics, Algorithms and Applications. Springer, 1st edition, 2008.

[37] P. Soille. Morphological image analysis. Springer-Verlag, 1999.

[38] S. Svensson and G. Sanniti di Baja. Simplifying curve skeletons in volume images. Computer Vision and Image Understanding, 90(3):242-257, 2003. 
[39] H. Talbot and L. Vincent. Euclidean skeletons and conditional bisectors. In Procs. VCIP'92, SPIE, volume 1818, pages 862-876, 1992.

[40] L. Vincent. Efficient computation of various types of skeletons. In Procs. Medical Imaging V, SPIE, volume 1445, pages 297-311, 1991.

[41] J.H.C. Whitehead. Simplicial spaces, nuclei and $m$-groups. Proceedings of the London Mathematical Society, 45(2):243-327, 1939. 